\title{
Financial Networks and Contagion
}

\section{By Matthew Elliott, Benjamin Golub, and Matthew O. Jackson*}

\begin{abstract}
We study cascades of failures in a network of interdependent financial organizations: how discontinuous changes in asset values (e.g., defaults and shutdowns) trigger further failures, and how this depends on network structure. Integration (greater dependence on counterparties) and diversification (more counterparties per organization) have different, nonmonotonic effects on the extent of cascades. Diversification connects the network initially, permitting cascades to travel; but as it increases further, organizations are better insured against one another's failures. Integration also faces trade-offs: increased dependence on other organizations versus less sensitivity to own investments. Finally, we illustrate the model with data on European debt cross-holdings. (JEL D85, F15, F34, F36, F65, G15, G32, G33, G38)
\end{abstract}

Globalization brings with it increased financial interdependencies among many kinds of organizations - governments, central banks, investment banks, firms, etc.that hold each other's shares, debts, and other obligations. Such interdependencies can lead to cascading defaults and failures, which are often avoided through massive bailouts of institutions deemed "too big to fail." Recent examples include the US government's interventions in AIG, Fannie Mae, Freddie Mac, and General Motors; and the European Commission's interventions in Greece and Spain. Although such bailouts circumvent the widespread failures that were more prevalent in the nineteenth and early twentieth centuries, they emphasize the need to study the risks created by a network of interdependencies. Understanding these risks is crucial to designing incentives and regulatory responses which defuse cascades before they are imminent.

In this paper we develop a general model that produces new insights regarding financial contagions and cascades of failures among organizations linked through a network of financial interdependencies. Organizations' values depend on each other-e.g., through cross-holdings of shares, debt, or other liabilities. If an

\footnotetext{
*Elliott: Division of Humanities and Social Sciences, California Institute of Technology, 1200 E. California Blvd., Pasadena, CA 91125 (e-mail: melliott@ caltech.edu); Golub: Department of Economics, Harvard University, Littauer Center, 1805 Cambridge St., Cambridge, MA 02138 (e-mail: ben.golub@ gmail.com); Jackson: Department of Economics, Stanford University, 579 Serra Mall, Stanford, CA 94305, the Santa Fe Institute, and CIFAR (e-mail: jacksonm@stanford.edu). Jackson gratefully acknowledges financial support from NSF grant SES-0961481 and grant FA9550-12-01-0411 from AFOSR and DARPA, and ARO MURI award No. W911NF-12-1-0509. All authors thank Microsoft Research New England Lab for research support. We thank Jean-Cyprien Héam, Scott Page, Gustavo Peralta, Ployplearn Ravivanpong, Alp Simsek, Alireza Tahbaz-Salehi, and Yves Zenou, as well as three referees and many seminar participants for helpful comments. The authors declare that they have no relevant or material financial interests that relate to the research described in this paper.

${ }^{\dagger}$ Go to http://dx.doi.org/10.1257/aer.104.10.3115 to visit the article page for additional materials and author disclosure statement(s).
} 
organization's value becomes sufficiently low, it hits a failure threshold at which it discontinuously loses further value; this imposes losses on its counterparties, and these losses then propagate to others, even those who did not interact directly with the organization initially failing. At each stage, other organizations may hit failure thresholds and also lose value discontinuously. Relatively small, and even organization-specific, shocks can be greatly amplified in this way. 1

In our model, organizations hold primitive assets (any factors of production or other investments) as well as shares in each other. ${ }^{2}$ The basic network we start with describes which organizations directly hold which others. Cross-holdings lead to a well-known problem of inflating book values, ${ }^{3}$ and so we begin our analysis by deriving a formula for a noninflated "market value" that any organization delivers to final investors outside the system of cross-holdings. This formula shows how each organization's market value depends on the values of the primitive assets and on any failure costs that have hit the economy. We can therefore track how asset values and failure costs propagate through the network of interdependencies. An implication of failures being complementary is that cascades occur in "waves" of dependencies. Although in practice these might occur all at once, it can be useful to distinguish the sequence of dependencies in order to figure out how they might be avoided. Some initial failures are enough to cause a second wave of organizations to fail. Once these organizations fail, a third wave of failures may occur, and so on. A variation on a standard algorithm $\rrbracket^{4}$ then allows us to compute the extent of these cascades by using the formula discussed above to propagate the failure costs at each stage and determine which organizations fail in the next wave. Policymakers can use this algorithm in conjunction with the market value formula to run counterfactual scenarios and identify which organizations might be involved in a cascade under various initial scenarios.

With this methodology in hand, our main results show how the probability of cascades and their extent depend on two key aspects of cross-holdings: integration and diversification. Integration refers to the level of exposure of organizations to each other: how much of an organization is privately held by final investors, and how much is cross-held by other organizations. Diversification refers to how spread out cross-holdings are: is a typical organization held by many others, or by just a few? Integration and diversification have different, nonmonotonic effects on the extent of cascades.

If there is no integration, then clearly there cannot be any contagion. As integration increases, the exposure of organizations to each other increases and so contagions become possible. Thus, on a basic level, increasing integration leads to increased exposure, which tends to increase the probability and extent of contagions. The countervailing effect here is that an organization's dependence on its own primitive

\footnotetext{
${ }^{1}$ The discontinuities incurred when an organization fails can include the cost of liquidating assets, the (temporary) misallocation of productive resources, as well as direct legal and administrative costs. Given that efficient investment or production can involve a variety of synergies and complementarities, any interruption in the ability to invest or pay for and acquire some factors of production can lead to discontinuously inefficient uses of other factors, or of investments. See Section IC for more details.

${ }^{2}$ We model cross-holdings as direct (linear) claims on values of organizations for simplicity, but the model extends to all sorts of debt and other contracts as discussed in Section 2 in the online Appendix.

${ }^{3}$ See Brioschi, Buzzacchi, and Colombo (1989) and Fedenia, Hodder, and Triantis (1994).

${ }^{4}$ This sort of algorithm is the obvious one for finding extreme points of a lattice, and so is standard in a variety of equilibrium settings. Ours is a variation on one from Eisenberg and Noe (2001).
} 
assets decreases as it becomes integrated. Thus, although integration can increase the likelihood of a cascade once an initial failure occurs, it can also decrease the likelihood of that first failure.

With regard to diversification, there are also trade-offs, but on different dimensions. Here the overall exposure of organizations is held fixed but the number of organizations cross-held is varied. With low levels of diversification, organizations can be very sensitive to particular others, but the network of interdependencies is disconnected and overall cascades are limited in extent. As diversification increases, a "sweet spot" is hit where organizations have enough of their cross-holdings concentrated in particular other organizations so that a cascade can occur, and yet the network of cross-holdings is connected enough for the contagion to be far-reaching. Finally, as diversification is further increased, organizations' portfolios are sufficiently diversified so that they become insensitive to any particular organization's failure.

Putting these results together, an economy is most susceptible to widespread financial cascades when two conditions hold. The first is that integration is intermediate: each organization holds enough of its own assets that the idiosyncratic devaluation of those assets can spark a first failure, and holds enough of other organizations for failures to propagate. The second condition is that organizations are partly diversified: the network is connected enough for cascades to spread widely, but nodes don't have so many connections that they are well-insured against the failure of any counterparty. Our analysis of these trade-offs includes both analytical results on a class of networks for which the dynamics of cascades are tractable, as well as simulation results on other random cross-holding networks.

In the simulations, we examine several important specific network structures. One is a network with a clique of large "core" organizations surrounded by many smaller "peripheral" organizations, each of which is linked to a core organization. This emulates the network of interbank loans. There we see a further nonmonotonicity in integration: if core organizations have low levels of integration, then the failure of some peripheral organization is contained, with only one core organization failing; if core organizations have middle levels of integration, then widespread contagions occur; if core organizations are highly integrated, then they become less exposed to any particular peripheral organization and more resistant to peripheral failures. A second model is one with concentrations of cross-holdings within sectors or other groups. As cross-holdings become more sector-specific, particular sectors become more susceptible to cascades, but widespread cascades become less likely. The level of segregation at which this change happens depends on diversification. With lower diversification, cascades disappear at lower rates of segregation-it takes less segregation to fragment the network and prevent cascades.

We also consider what a regulator or government might do to mitigate the possibility of cascades of failures. Preventing a first failure prevents the potential ensuing cascade of failures, and it might be hoped that a clever reallocation of cross-holdings could achieve this. Unfortunately, we show that any fair exchange of cross-holdings or assets involving the organization most at risk of failing makes that organization more likely to fail at some asset prices close to the current asset prices. Making the system unambiguously less susceptible to a first failure necessitates bailing out the organization most at risk of failing. 
Finally, we illustrate the model in the context of cross-holdings of European debt.

While there is a growing literature on networks of interdependencies in financial markets, ${ }^{5}$ our methodology and results are different from any that we are aware of, especially the results on nonmonotonicities in cascades due to integration and diversification.

An independent study by Acemoglu, Ozdaglar, and Tahbaz-Salehi (2012) —as well as related earlier studies of Gouriéroux, Héam, and Monfort (2012) and Gai and Kapadia (2010) — are the closest to ours. ${ }^{6}$ They each examine how shocks propagate through a network based on debt holdings or interbank lending, where shocks lead an organization to pay only a portion of its debts. They are also interested in how shocks propagate as a function of network architecture. However, beyond the basic motivation and focus on the network propagation of shocks, the studies are quite different and complementary. The main results of Acemoglu, Ozdaglar, and Tahbaz-Salehi (2012) characterize the best and worst networks from a social planner's perspective. For moderate shocks a perfectly diversified pattern of holdings is optimal, while for very large shocks perfectly diversified holdings become the worst possible. 7 Our focus is on the complementary question of what happens for intermediate shocks and for a variety of networks. To this end, we consider a class of random networks and ask how the consequences of a given moderate shock depend on diversification and integration. The results highlight that intermediate levels of diversification and integration can be the most problematic.

Gai and Kapadia (2010) made two observations. First: rare, large shocks may have extreme consequences when they occur-a point elaborated upon in the subsequent literature discussed above. Second, a shock of a given magnitude may have very different consequences depending on where in the network it hits and on the average connectivity of the network. Gai and Kapadia develop these points in a standard model of epidemics in which the network is characterized by its degree distribution. An innovation of our model is to go beyond the degree distribution of a network and calculate equilibrium (fixed-point) values and interdependencies for organizations. Doing so allows us to distinguish an important dimension of financial networks: integration, which can be varied independently of diversification. Building on that, we show how diversification and integration each affect the ingredients of financial cascades - and the final outcomes - in different and nonmonotonic ways. In doing so, we recover, as a special case, Gai and Kapadia's observation that cascades can

\footnotetext{
${ }^{5}$ For example, see Rochet and Tirole (1996); Kiyotaki and Moore (1997); Allen and Gale (2000); Eisenberg and Noe (2001); Upper and Worms (2004); Cifuentes, Ferrucci, and Shin (2005); Leitner (2005); Allen and Babus (2009); Lorenz, Battiston, and Schweitzer (2009); Gai and Kapadia (2010); Wagner (2010); Billio et al. (2012); Demange (2012); Diebold and Yilmaz (2011); Dette, Pauls, and Rockmore (2011); Gai, Haldane, and Kapadia (2011); Greenwood, Landier, and Thesmar (2012); Ibragimov, Jaffee, and Walden (2011); Upper (2011); Acemoglu et al. (2012); Allen, Babus, and Carletti (2012); Cohen-Cole, Patacchini, and Zenou (2012); Gouriéroux, Héam, and Monfort (2012); Alvarez and Barlevy (2013); Glasserman and Young (2013); and Gofman (2013).

${ }^{6}$ Cabrales, Gottardi, and Vega-Redondo (2013) study the trade-off between the risk-sharing enabled by greater interconnection and the greater exposure to cascades resulting from larger components in the financial network. Their focus is also on some benchmark networks (minimally connected and complete ones) and they examine which ones are best for different distributions of shocks. Again, our work is complementary not only in terms of distinguishing diversification and integration but also analyzing comparative statics for intermediate network structures and finding nonmonotonicities there.

${ }^{7}$ Shaffer (1994) also identifies a trade-off between risk sharing and systemic failures. While diversified portfolios reduce risk, they also result in organizations holding similar portfolios and a system susceptible to simultaneous failures. See also Ibragimov, Jaffee, and Walden (2011) and Allen, Babus, and Carletti (2012).
} 
be nonmonotonic in connectivity. ${ }^{8}$ But we also gain key new results on when and how the "danger zone" of intermediate diversification can be blunted by changing the level of integration in the system. Finally, we study how the integration of a financial network interacts with a core-periphery structure and with segregation, and other correlation structures.

\section{The Model and Determining Organizations' Values with Cross-Holdings}

\section{A. Primitive Assets, Organizations, and Cross-Holdings}

There are $n$ organizations (e.g., countries, banks, or firms) making up a set $N=\{1, \ldots, n\}$.

The values of organizations are ultimately based on the values of primitive assets

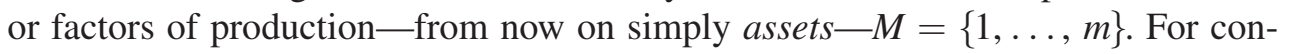
creteness, a primitive asset may be thought of as a project that generates a net flow of cash over time. ${ }^{9}$ The present value (or market price) of asset $k$ is denoted $p_{k}$. Let $D_{i k} \geq 0$ be the share of the value of asset $k$ held by (i.e., flowing directly into) organization $i$ and let $\mathbf{D}$ denote the matrix whose $(i, k)$ th entry is equal to $D_{i k}$. (Analogous notation is used for all matrices.)

An organization can also hold shares of other organizations. For any $i, j \in N$ the number $C_{i j} \geq 0$ is the fraction of organization $j$ owned by organization $i$, where $C_{i i}=0$ for each $i .^{10}$ The matrix $\mathbf{C}$ can be thought of as a network in which there is a directed link from $i$ to $j$ if $i$ owns a positive share of $j$, so that $C_{i j}>0 .{ }^{11}$ Paths in this network are called ownership paths. We also sometimes work with a graphical representation of $\mathbf{C}$ where directed links point in the opposite direction, the direction in which value (and loss of value) flows. We call the paths in that network cascade paths.

After all these cross-holding shares are accounted for, there remains a share $\hat{C}_{i i}:=1-\sum_{j \in N} C_{j i}$ of organization $i$ not owned by any organization in the system-a share assumed to be positive. ${ }^{12}$ This is the part that is owned by outside shareholders of $i$, external to the system of cross-holdings. The off-diagonal entries of the matrix $\hat{\mathbf{C}}$ are defined to be 0 .

Cross-holdings are modeled as linear dependencies in this paper, and we now briefly discuss the interpretation of this. We view the functional form as an approximation of debt contracts around and below organizations' failure thresholds- the

\footnotetext{
${ }^{8}$ In different settings, Cifuentes, Ferrucci, and Shin (2005) and Gofman (2013) also find that cascades can be nonmonotonic in connectivity.

${ }^{9}$ The primitive assets could be more general factors: prices of inputs, values of outputs, the quality of organizational know-how, investments in human capital, etc. To keep the exposition simple, we model these as abstract investments and assume that net positions are nonnegative in all assets.

${ }^{10}$ It is possible to instead allow $C_{i i}>0$, which leads to some straightforward adjustments in the derivations that follow; but one needs to be careful in interpreting what it means for an organization to have cross-holdings in itself — which effectively translates into a form of private ownership.

${ }^{11}$ Some definitions: a path from $i_{1}$ to $i_{\ell}$ in a matrix $\mathbf{M}$ is a sequence of distinct nodes $i_{1}, i_{1}, \ldots, i_{\ell}$ such that $M_{i_{r+1} i_{r}}>0$ for each $r \in\{1,2, \ldots, \ell-1\}$. A cycle is a sequence of (not necessarily distinct) nodes $i_{1}, i_{1}, \ldots, i_{\ell}$ such that $M_{i_{r+1} i_{r}}>0$ for each $r \in\{1,2, \ldots, \ell-1\}$ and $M_{i_{1} i_{r}}>0$.

${ }^{12}$ This assumption ensures that organizations' market values (discussed below) are well defined. It is slightly stronger than necessary. It would suffice to assume that, for every organization $i$, there is some $j$ such that $\hat{C}_{j j}>0$ and there is an ownership path from $j$ to $i$. An organization with $\hat{C}_{i i}=0$ would essentially be a holding company, and the important aspect is to have an economy where there are at least some organizations that are not holding companies and some outside shareholders that no organizations have claims on.
} 
region of organizations' values that are important whenever one's failure causes another to fail. In this region, under most bankruptcy procedures ${ }^{13}$ there is linear rationing in how much of the debt is paid back. Some organizations may be far from their failure thresholds, and for those, others' changes in value have a smaller effect on the risk of failure. The linear model can incorporate both of these effects through the slope parameters in the cross-holdings matrix; this is discussed in detail in Section IE, as well as Section 2 of the online Appendix. Of course, this is a crude approximation, but allows a tractable analysis of cross-dependencies, and provides basic insights that should still be useful when nonlinearities are addressed in detail. More generally, cross-holdings can involve all sorts of contracts; any liability in the form of some payment that is due could be included. ${ }^{14}$ Directly modeling other sorts of contracting between organizations would complicate the analysis, and so we focus on this formulation for now to illustrate the basic issues. Section 2 in the online Appendix discusses extending the model to more general liabilities.

\section{B. Values of Organizations: Accounting and Adjusting for Cross-Holdings}

In a setting with cross-holdings, there are subtleties in determining the "fair market" value of an organization, and the real economic costs of organizations' failures. Doing the accounting correctly is essential to analyzing cascades of failure. The basic framework for the accounting was developed by Brioschi, Buzzacchi, and Colombo (1989) and Fedenia, Hodder, and Triantis (1994). In this section, we briefly review the accounting and the key valuation equations in the absence of failure costs. In ensuing sections, we incorporate failures and associated discontinuities.

The equity or book value $V_{i}$ of an organization $i$ is the total value of its sharesthose held by other organizations as well as those held by outside shareholders. This is equal to the value of organization $i$ 's primitive assets plus the value of its claims on other organizations:

$$
V_{i}=\sum_{k} D_{i k} p_{k}+\sum_{j} C_{i j} V_{j}
$$

Equation (1) can be written in matrix notation as

$$
\mathbf{V}=\mathbf{D p}+\mathbf{C V}
$$

and solved to yield ${ }^{15}$

$$
\mathbf{V}=(\mathbf{I}-\mathbf{C})^{-1} \mathbf{D p}
$$

\footnotetext{
${ }^{13}$ A richer model would include priority classes, but the basic issues that we address in the simplified model should still appear in such a richer model.

${ }^{14}$ In essence, our modeling is a reduced form that aggregates all effects into a linear dependence of each organization on others, allowing for a discontinuous loss at a critical organization value. In cases where organizations can short-sell other organizations, or hold options or other derivatives that appreciate in value when another organization falls in value, some of our lattice results (discussed in Sections IF and IIB) would no longer hold. That is an interesting topic for further research.

${ }^{15}$ Under the assumption that each column of $\mathbf{C}$ sums to less than 1 (which holds by our assumption of nonzero outside holdings in each organization), the inverse $(\mathbf{I}-\mathbf{C})^{-1}$ is well-defined and nonnegative (Meyer 2000, section 7.10).
} 
Adding up equation (1) across organizations (and recalling that each column of $\mathbf{D}$ adds up to 1) shows that the sum of the $V_{i}$ exceeds the total value of primitive assets held by the organizations. Essentially, each dollar of net primitive assets directly held by organization $i$ contributes one dollar to the equity value of organization $i$, but then is also counted partially on the books of all the organizations that have an equity stake in $i .^{16}$

As argued by both Brioschi, Buzzacchi, and Colombo (1989) and Fedenia, Hodder, and Triantis (1994), the ultimate (noninflated) value of an organization to the economy — what we call the "market" value-is well-captured by the equity value of that organization that is held by its outside investors. This value captures the flow of real assets which accrues to final investors of that organization. The market value, which we denote by $v_{i}$, is equal to $\hat{C}_{i i} V_{i}$, and therefore: ${ }^{17}$

$$
\mathbf{v}=\hat{\mathbf{C}} \mathbf{V}=\hat{\mathbf{C}}(\mathbf{I}-\mathbf{C})^{-1} \mathbf{D p}=\mathbf{A D p}
$$

We refer to $\mathbf{A}=\hat{\mathbf{C}}(\mathbf{I}-\mathbf{C})^{-1}$ as the dependency matrix. It is reminiscent of Leontief's (1951) input-output analysis. Equation (3) shows that the value of an organization can be represented as a sum of the values of its ultimate claims on primitive assets, with organization $i$ owning a share $A_{i j}$ of $j$ 's direct holdings of primitive assets. This is the portfolio of underlying assets an outside investor would hold to replicate the returns generated by holding organization $i$. To see this, suppose each organization fully owns exactly one proprietary asset, so that $m=n$ and $\mathbf{D}=\mathbf{I}$. In this case, $A_{i j}$ describes the dependence of $i$ 's value on $j$ 's proprietary asset. It is reassuring that $\mathbf{A}$ is column-stochastic, so that indeed the total values of all organizations add up to the total values of all underlying assets-for all $j \in N$, we have ${ }^{18}$

$$
\sum_{i \in N} A_{i j}=1
$$

\footnotetext{
${ }^{16}$ This initially counterintuitive feature is discussed in detail by French and Poterba (1991) and Fedenia, Hodder, and Triantis (1994).

${ }^{17}$ A way to double-check this equation is to derive the market value of an organization from the book value of its underlying assets and cross-holdings less the part of its book value promised to other organizations in cross-holdings:

$$
v_{i}=\sum_{j} C_{i j} V_{j}-\sum_{j} C_{j i} V_{i}+\sum_{k} D_{i k} p_{k}
$$
}

or

$$
\mathbf{v}=\mathbf{C V}-(\mathbf{I}-\hat{\mathbf{C}}) \mathbf{V}+\mathbf{D p}=(\mathbf{C}-(\mathbf{I}-\hat{\mathbf{C}})) \mathbf{V}+\mathbf{D p} .
$$

Substituting for the book value $\mathbf{V}$ from (2), this becomes

$$
\mathbf{v}=(\mathbf{C}-\mathbf{I}+\hat{\mathbf{C}})(\mathbf{I}-\mathbf{C})^{-1} \mathbf{D p}+\mathbf{D p}=(\mathbf{C}-\mathbf{I}+\hat{\mathbf{C}}+(\mathbf{I}-\mathbf{C}))(\mathbf{I}-\mathbf{C})^{-1} \mathbf{D p}=\mathbf{A D p} .
$$

\footnotetext{
${ }^{18}$ This can be seen by defining an augmented system in which there is a node corresponding to each organization's external investors and noting that, under our assumptions, the added nodes are the only absorbing states of the Markov chain corresponding to the system of asset flows. Column $j$ of $\mathbf{A}$ describes how the proprietary assets entering at node $j$ are shared out among the external absorbing nodes. Since all the flow must end up at some external absorbing node, A must be column-stochastic.
} 


\section{Discontinuities in Values and Failure Costs}

An important part of our model is that organizations can lose productive value in discontinuous ways if their values fall below certain critical thresholds. These discontinuities can lead to cascading failures and also the presence of multiple equilibria.

There are many sources of such discontinuities. For example, if an airline can no longer pay for fuel, then its planes may be forced to sit idle (as happened with Spanair in February of 2012), which leads to a discontinuous drop in revenue in response to lost bookings, and so forth. If a country or firm's debt rating is downgraded, it often experiences a discontinuous jump in its cost of capital. Dropping below a critical value might also involve bankruptcy proceedings and legal costs. Broadly, many of these discontinuities stem from an illiquidity which then leads to an inefficient use of assets. Indeed, given that efficient production can involve a variety of synergies and complementarities, any interruption in the ability to pay for and acquire some factors of production can lead to discontinuously inefficient uses of other factors, or of investments. One detailed and simple microfoundation is laid out in Section IE below.

If the value $v_{i}$ of an organization $i$ falls below some threshold level $\underline{v}_{i}$, then $i$ is said to fail and incurs failure costs $\beta_{i}(\mathbf{p})$. ${ }^{19}$ These failure costs are subtracted from a failing organization's cash flow. They can represent the diversion of cash flow towards dealing with the failure or a reduction in the returns generated by proprietary assets. Either way, this introduces critical nonlinearities - indeed, discontinuities - into the system.

We base failure costs on the (market) value of an organization $v_{i}$, and not the book or equity value, $V_{i}$. This captures the idea that failure occurs when an organization has difficulties or disruptions in operating, and the artificial inflation in book values that accompanies cross-holdings is irrelevant in avoiding a failure threshold. ${ }^{20}$ Nonetheless, the model could instead make failures dependent upon the book values $V_{i}$, in cases where cash flows relate to book values. Nothing qualitative would change in what follows, as the critical ingredients of thresholds of discontinuities and cascades that depend on cross-holdings would still all be present, just with different trigger points.

Let us say a few words about the relative sizes of these discontinuities. Recent work has estimated the cost of default to average 21.7 percent of the market value of an organization's assets (with substantial variation-see Davydenko, Strebulaev, and Zhao 2012, as well as James 1991).21 It might be hoped that organizations will reduce the scope for cascades of failures by minimizing their failure costs and reducing the threshold values at which they fail. In fact, as we show in the online Appendix (Section 3), financial networks can create moral hazard and favor the opposite outcome. As discussed in Leitner (2005) and Rogers and Veraart (2013),

\footnotetext{
${ }^{19}$ The argument $\mathbf{p}$ reflects that these costs can depend on the values of underlying assets, as would be the case when these are liquidated for a fraction of their former value. See Section IE for more detail.

${ }^{20}$ For example, if the failure threshold were based on book values, then two organizations about to fail would be able to avoid failure by exchanging cross-holdings and inflating their book values.

${ }^{21}$ Capping the failure costs is not important for our model, but they could easily be capped at $\underline{v}_{i}$ or $(\mathbf{D p})_{i}$ or some other natural level.
} 
counterparties have incentives to bail out a failing organization ${ }^{22}$ to avoid (indirectly) incurring failure costs. To improve its bargaining position in negotiating for such aid, an organization may then want to increase its failure costs and make its failure more likely. Nevertheless, although default costs can be large both absolutely and relative to the value of an organization's assets (e.g., the size of the recent Greek write-down in debt, or the fire sale of Lehman Brothers' assets), it can also be that smaller effects snowball. Given that a major recession in an economy is only a matter of a change of a few percentage points in its growth rate, when contagions are far-reaching, the particular drops in value of any single organization need not be very large in order to have a large effect on the economy. We develop this observation further in Section IIA.

\section{Including Failure Costs in Market Values}

The valuations in (2) and (3) have analogs when we include discontinuities in value due to failures. The discontinuous drop imposes a cost directly on an organization's balance sheet, and so the book value of organization $i$ becomes:

$$
V_{i}=\sum_{j \neq i} C_{i j} V_{j}+\sum_{k} D_{i k} p_{k}-\beta_{i} I_{v_{i}<\underline{v}}
$$

where $I_{v_{i}<\underline{v}_{i}}$ is an indicator variable taking value 1 if $v_{i}<\underline{v}_{i}$ and value 0 otherwise.

This leads to a new version of (2):

$$
\mathbf{V}=(\mathbf{I}-\mathbf{C})^{-1}(\mathbf{D} \mathbf{p}-\mathbf{b}(\mathbf{v}, \mathbf{p}))
$$

where $b_{i}(\mathbf{v}, \mathbf{p})=\beta_{i}(\mathbf{p}) I_{v_{i}<\underline{v}_{i}} \cdot{ }^{23}$ Correspondingly, (3) is re-expressed as

$$
\mathbf{v}=\hat{\mathbf{C}}(\mathbf{I}-\mathbf{C})^{-1}(\mathbf{D p}-\mathbf{b}(\mathbf{v}))=\mathbf{A}(\mathbf{D p}-\mathbf{b}(\mathbf{v}, \mathbf{p})) .
$$

An entry $A_{i j}$ of the dependency matrix describes the proportion of $j$ 's failure costs that $i$ bears when $j$ fails as well as $i$ 's claims on the primitive assets that $j$ directly holds. If organization $j$ fails, thereby incurring failure costs of $\beta_{j}$, then $i$ 's value will decrease by $A_{i j} \beta_{j}$.

\section{E. A Simple Microfoundation}

To help fix ideas, we discuss one simple microfoundation-among many-of the model and the value equations provided above.

Organizations are owner-operated firms. For simplicity, let each firm have a single proprietary asset: an investment project that generates a return. Our model is then simplified to the case $m=n$ and $\mathbf{D}=\mathbf{I}$. Firms have obligations to each other: for instance, promised payments for inputs or other intermediate goods. These

\footnotetext{
${ }^{22}$ For example, in the form of a debt write-down.

${ }^{23}$ The number $b_{i}(\mathbf{v}, \mathbf{p})$ reflects realized failure costs, and is zero when failure does not occur. It always depends on the asset values through the indicator $I_{v_{i}<v}$, but the bankruptcy costs $\beta_{i}$ may depend on underlying asset values, p. See Section IE for an example. We suppress the argument $\mathbf{p}$ when it is not essential.
} 
obligations comprise the cross-holdings. Once a firm's value no longer covers the full promised value of its payments, all creditor organizations-who are of equal seniority-are rationed in proportion to $V_{i}$, with organization $j$ claiming $C_{i j} V_{i}$ of $i$ 's value. Thus, even though the obligations might initially be in the form of debt, the relevant scenario for our cascades - and the one the model focuses on-is one in which the full promised amounts cannot be met by the organizations. This is a regime of "orderly write-downs" in which creditors are willing to take a fraction of the face value they are owed. Thus, the values of cross-holdings are simply linear in $V_{i}$, as in our equations. (Section 2 in the online Appendix illustrates this in detail.)

The value left to the owner-operators is $v_{i}=\hat{C}_{i i} V_{i}$. While the firm continues to operate, this amount must cover return on capital, wages, benefits, and pension obligations for the owner-operators. ${ }^{24}$ The share $\hat{C}_{i i}$ can be thought of as all of the stock or equity held in the firm, while the $C_{i j}$ 's are payment obligations from the firm to other firms. The $\hat{C}_{i i}$ residual shares correspond to the control rights of the firm, while the $C_{i j}$ 's simply represent obligations to other creditors. If the value left to the owner-operators/shareholders is sufficiently low (below some outside option value of their time or effort), they may choose to cease operations. ${ }^{25}$ Indeed, we posit that there is a critical threshold $\underline{v}_{i}$ such that if the value available to the owner-operator falls below it, he or she chooses to cease operations and to liquidate the asset. In other words, once $v_{i}<\underline{v}_{i}$ the asset is liquidated.

Liquidation is irreversible and total: a firm cannot partially liquidate its proprietary asset. Liquidation is also costly: if $i$ liquidates its proprietary asset, it incurs a loss of $\lambda_{i}$ cents on the dollar. ${ }^{26}$ In terms of our model, $\beta_{i}(\mathbf{p})=\lambda_{i} p_{i}$. Recalling that $b_{i}(\mathbf{v}, \mathbf{p})=\beta_{i}(\mathbf{p}) I_{v_{i}<\underline{v}_{i}}$, it follows that

$$
\mathbf{v}=\mathbf{A}(\mathbf{p}-\mathbf{b}(\mathbf{v}, \mathbf{p}))
$$

\section{F. Equilibrium Existence and Multiplicity}

A solution for organization values in equation (5) is an equilibrium set of values, and encapsulates the network of cross-holdings in a clean and powerful form, building on the dependency matrix $\mathbf{A}$.

There always exists a solution-and there can exist multiple solutions - to the valuation equation (multiple vectors $\mathbf{v}$ satisfying (5)) in the presence of the discontinuities. In fact, the set of solutions forms a complete lattice. ${ }^{27}$

There are two distinct sources of equilibrium multiplicity. First, taking other organizations' values and the values of underlying assets as fixed and given, there can be multiple possible consistent values of organization $i$ that solve equation (5). There may be a value of $v_{i}$ satisfying equation (5) such that $1_{v_{i}<\underline{v}_{i}}=0$ and another value of $v_{i}$ satisfying equation (5) such that $1_{v_{i}<\underline{v}_{i}}=1$, even when all other prices and values

\footnotetext{
${ }^{24}$ Indirectly, the value $v_{i}$ includes the cross-holdings that firm $i$ has in others: that is, accounts receivable that can be used to meet payroll and other obligations.

${ }^{25}$ This can happen for various reasons. For example, in the case of Spanair, there was too little money to cover wages, fuel, and other basic maintenance costs, and the airline was forced to cease operations. It could also be that the owners no longer view it worthwhile to continue to devote efforts to this investment project.

${ }^{26}$ These losses involve time that the asset is left idle, costs of assessing values and holding sales of assets, costs of moving assets to another production venue, and loss of firm-specific capital and knowledge.

${ }^{27}$ This holds by a standard application of Tarski's fixed point theorem, as failures are complements.
} 
are held fixed. This source of multiple equilibria corresponds to the standard story of self-fulfilling bank runs (see classic models such as Diamond and Dybvig 1983). The second source of multiple equilibria is the interdependence of the values of the organizations: the value of $i$ depends on the value of organization $j$, while the value of organization $j$ depends on the value of organization $i$. There might then be two consistent valuation vectors for $i$ and $j$ : one in which both $i$ and $j$ fail, and another in which both $i$ and $j$ remain solvent. This second source of multiple equilibria is different from the individual bank-run concept, as here organizations fail because people expect other organizations to fail, which then becomes self-fulfilling.

In what follows, we typically focus on the best-case equilibrium, in which as few organizations as possible fail. ${ }^{28}$ This allows us to isolate sources of necessary cascades, distinct from self-fulfilling sorts of failure, which have already been studied in the sunspot and bank-run literatures. When we do discuss multiple equilibria, we will consider only the second novel source of multiplicity-multiplicity due to interdependencies between organizations - rather than the well-known phenomenon of a bank run on a single organization. With suitable regularity conditions (so that other equilibria are appropriately stable in some range of parameters), the results presented below should have analogs applying to other equilibria, including the worst-case equilibrium.

\section{G. Measuring Dependencies}

The dependency matrix $\mathbf{A}$ takes into account all indirect holdings as well as direct holdings. The central insights of the paper are derived using this matrix. In this section we identify some useful properties of the dependency matrix $\mathbf{A}$ and explore its relation to direct cross-holdings $\mathbf{C}$.

An Example.-To see how the dependency matrix A and direct cross-holdings matrix $\mathbf{C}$ might differ, consider the following example. Suppose there are two organizations, $i=1,2$, each of which has a 50 percent stake in the other organization. The associated cross-holdings matrix $\mathbf{C}$ and the dependency matrix $\mathbf{A}$ are as follows. (Recall that $\hat{C}_{i i}$ is equal to 1 minus the sum of the entries in column $i$ of $\mathbf{C}$.)

$$
\mathbf{C}=\left(\begin{array}{cc}
0 & 0.5 \\
0.5 & 0
\end{array}\right) \quad \hat{\mathbf{C}}=\left(\begin{array}{cc}
0.5 & 0 \\
0 & 0.5
\end{array}\right) \quad \mathbf{A}=\hat{\mathbf{C}}(\mathbf{I}-\mathbf{C})^{-1}=\left(\begin{array}{cc}
\frac{2}{3} & \frac{1}{3} \\
\frac{1}{3} & \frac{2}{3}
\end{array}\right)
$$

In this simple example, we can already see that direct claims - as captured by $\mathbf{C}$ and $\hat{\mathbf{C}}$ - can differ quite substantially from the ultimate value dependencies described by A. First, even though organization 1's shareholders have a direct claim on 50 percent of its value, they are ultimately entitled to more than this — as they also have some claims on the value of organization 2 , which includes part of the value of

\footnotetext{
${ }^{28}$ As discussed in Section IIB, in this best-case equilibrium no organization fails that does not also fail in all other equilibria.
} 
organization 1. Second, the ultimate dependence of each organization on the other is smaller than what is apparent from $\mathbf{C}$, by the fact that value is conserved. ${ }^{29}$

Although A can differ substantially from the direct holdings captured by $\mathbf{C}+\hat{\mathbf{C}}$, some general statements can be made about the differences.

LEMMA 1: $\hat{C}_{i i}$ is a lower bound on $A_{i i}$, and $A_{i i}$ can be much larger than $\hat{C}_{i i}$.

(i) $\frac{A_{i i}}{\hat{C}_{i i}} \geq 1$ for each $i$, with equality if and only if there are no cycles of crossholdings (i.e., directed ownership cycles in $\mathbf{C}$ ) that include $i$.

(ii) For any $n \geq 2$, there exists a sequence of $n$-by- $n$ matrices $\left(\mathbf{C}^{(\ell)}\right)$ such that $\frac{A_{i i}^{(\ell)}}{\hat{C}_{i i}^{(\ell)}} \rightarrow \infty$ for all $i$.

The magnitudes of the terms on the main diagonal of $\mathbf{A}$ turn out to be critical for determining whether and to what extent failures cascade (Section IIA) and the size of a moral hazard problem we discuss in the online Appendix (Section 3). Lemma 1 demonstrates that the lead diagonal of $\mathbf{A}$ can be larger than the lead diagonal of $\hat{\mathbf{C}}$, but can never be smaller. The potential for a large divergence comes from the fact that sequences of cross-holdings can involve cycles ( $i$ holds $j$, who holds $k$, who holds $\ell, \ldots$, who holds $i$ ), so that $i$ can end up with a higher dependency on its own assets than indicated by looking only at its outside investors' direct holdings $\left(\hat{C}_{i i}\right)$.

\section{H. Avoiding a First Failure}

Before moving on to our main results regarding diversification and integration, we provide a result which uses our model to show that there are necessarily trade-offs in preventing the spark that ignites a cascade. Any fair trades of cross-holdings and assets that help an organization avoid failure in some circumstances must make it vulnerable to failure in some new circumstances. This is a sort of "no-free-lunch" result for avoiding first failures.

To state this result, it is helpful to introduce some notation. We write organization $i$ 's value assuming no failures at asset prices $\mathbf{p}$, cross-holdings $\mathbf{C}$, and direct holdings $\mathbf{D}$ as $v_{i}(\mathbf{p}, \mathbf{C}, \mathbf{D})$. An organization $i$ is closest to failing at positive asset prices $\mathbf{p}$, cross-holdings $\mathbf{C}$, and direct holdings $\mathbf{D}$ if there exists a (necessarily unique) $\lambda>0$ such that at asset prices $\lambda \mathbf{p}$, organization $i$ is about to fail, $v_{i}(\lambda \mathbf{p}, \mathbf{C}, \mathbf{D})=\underline{v}_{i}$, while all other organizations are solvent, $v_{j}(\lambda \mathbf{p}, \mathbf{C}, \mathbf{D})>\underline{v}_{j}$ for $j \neq i$. Define $\mathbf{q}(\mathbf{p}, \mathbf{C}, \mathbf{D}):=\lambda \mathbf{p}$.

Before stating the result we also introduce the concept of fair trades. ${ }^{30}$ Fair trades are exchanges of cross-holdings or underlying assets which leave the (market) values of the organizations unchanged at current asset prices. ${ }^{31}$ More precisely, the matrices $(\mathbf{C}, \mathbf{D})$ and $\left(\mathbf{C}^{\prime}, \mathbf{D}^{\prime}\right)$ are said to be related by a fair trade at $\mathbf{p}$ if $\mathbf{v}=\mathbf{v}^{\prime}$, where

\footnotetext{
${ }^{29} \mathbf{A}$ further (starker) illustration of how $\mathbf{A}$ and $\mathbf{C}$ can differ is available in the online Appendix (Section 1).

${ }^{30}$ This definition takes prices of assets $(\mathbf{p})$ as given, but not the prices of organizations, valuing them based on their holdings. It does not incorporate the potential impact of failures of organizations on their values. Thus it is a benchmark that abstracts away from the failure costs, which is the right benchmark for the exercise of seeing the impact of trades on first failures.

${ }^{31}$ So, absent failure, the values of organizations are the same before and after fair trades.
} 
$\mathbf{v}=\mathbf{A p}$ and $\mathbf{v}^{\prime}=\mathbf{A} \mathbf{p}$; the matrix $\mathbf{A}^{\prime}$ is computed as in (5), with $\mathbf{C}^{\prime}$ and $\mathbf{D}^{\prime}$ playing the roles of $\mathbf{C}$ and $\mathbf{D} .^{32}$

PROPOSITION 1: Suppose an organization $i$ is closest to failing at asset prices $\mathbf{p}>\mathbf{0}$, cross-holdings $\mathbf{C}$, and direct holdings $\mathbf{D}$. Consider new cross-holdings and direct holdings $\mathbf{C}^{\prime}$ and $\mathbf{D}^{\prime}$ resulting from a fair trade at $\mathbf{p}$ such that row $i$ of $\mathbf{A}^{\prime}$ is different from that of $\mathbf{A}$. Then, for any $\varepsilon>0$, there is a $\mathbf{p}^{\prime}$ within an $\varepsilon$-neighborhood of $\mathbf{q}(\mathbf{p}, \mathbf{C}, \mathbf{D})>0,{ }^{33}$ such that $i$ fails at prices $\mathbf{p}^{\prime}$ after the fair trade but not before: $v_{i}\left(\mathbf{p}^{\prime}, \mathbf{C}^{\prime}, \mathbf{D}^{\prime}\right)<\underline{v}_{i}<v_{i}\left(\mathbf{p}^{\prime}, \mathbf{C}, \mathbf{D}\right)$.

It is conceivable that if an organization is at risk of eventual failure but not imminent failure, there could exist some fair trades that would unambiguously make that organization safer: prone to failure at a smaller set of prices. An organization might hedge a particular risk. Proposition 1 shows that, at least when it comes to saving the most vulnerable organization, there are always trade-offs: new holdings that avoid failure at one set of prices make failure more likely at another set of nearby prices. So, to fully avoid a failure (at nearby prices) once it is imminent requires some unfair trades or external infusion of capital.

\section{Cascades of Failures: Definitions and Preliminaries}

In order to present our main results, we need to first provide some background results and definitions regarding how the model captures cascades, which we present in this section. These preliminaries outline how failures cascade and become amplified, a simple algorithm for identifying the waves of failures in a cascade, and our distinction between diversification and integration.

\section{A. Amplification through Cascades of Failures}

A relatively small shock to even a small organization can have large effects by triggering a cascade of failures. The following example illustrates this. For simplicity, suppose that organization 1 has complete ownership of a single asset with value $p_{1}$. Suppose that $\mathbf{p}^{\prime}$ differs from $\mathbf{p}$ only in the price of asset 1 , and such that $p_{1}^{\prime}<p_{1}$. Finally, suppose $v_{1}(\mathbf{p})>\underline{v}_{1}>v_{1}\left(\mathbf{p}^{\prime}\right)$, so that 1 fails after the shock changing asset values from $\mathbf{p}$ to $\mathbf{p}^{\prime}$. Beyond the loss in value due to the decrease in the value of asset 1 , organization 2's value also decreases by a term arising from 1's failure cost, $A_{21} \beta_{1}$ (recall (5)). If organization 2 also fails, organization 3 absorbs part of both failure costs: $A_{31} \beta_{1}+A_{32} \beta_{2}$, and so organization 3 may fail too, and so forth. With each failure, the combined shock to the value of each remaining solvent organization increases and organizations that were further and further from failure before the initial shock can get drawn into the cascade. If, for example, the first $K$ organizations end up failing in the cascade, the cumulative failure costs to the economy are $\beta_{1}+\cdots+\beta_{K}$, which can greatly exceed the drop in asset value that precipitated the cascade.

\footnotetext{
${ }^{32}$ We show in Section 3.1 of the online Appendix that there are circumstances under which organizations may have incentives to undertake "unfair" trades because of the failure costs.

${ }^{33}$ That is, $\mathbf{p}^{\prime}$ such that $\left\|\mathbf{p}^{\prime}-\mathbf{q}(\mathbf{p}, \mathbf{C}, \mathbf{D})\right\|_{\infty}<\varepsilon$, where $\|\cdot\|_{\infty}$ denotes the sup-norm.
} 


\section{B. Who Fails in a Cascade?}

A first step towards understanding how susceptible a system is to a cascade of failures, and how extensive such a cascade might be, is to identify which organizations fail following a shock. Again, we focus on the best-case equilibrium. ${ }^{34}$ Studying the best-case equilibrium following a shock identifies the minimal possible set of organizations that could fail. (Results for the worst-case equilibrium are easy analogs, identifying the maximal possible set of organizations that fail.)

Identifying Who Fails When.-To understand how and when failures cascade we need to better understand when a fall in asset prices will cause an initial failure and whether the first failure will result in other failures. Utilizing the dependency matrix A, for each organization $i$ we can identify the boundary in the space of underlying asset prices below which organization $i$ must fail, assuming no other organization has failed yet. We can also identify how the failure of one organization affects the failure boundaries of other organizations and so determine when cascades will occur and who will fail in those cascades. We begin with an example that illustrates these ideas very simply, and then develop the more general analysis.

Example Continued.-Let us return to the example introduced in Section IG, taking $\mathbf{D}=\mathbf{I}$, so each organization owns one proprietary asset. We suppose that organization $i$ fails when its value falls below 50 and upon failing incurs failure costs of 50 . Organization $i$ therefore fails when $\frac{2}{3} p_{i}+\frac{1}{3} p_{j}<50$. Panel A of Figure 1 shows the failure frontiers for the two organizations. When asset prices are above both failure frontiers, neither organization fails in the best-case equilibrium outcome. One object that we study is the boundary between this region and the region in which at least one organization fails in all equilibria. We call this boundary the first failure frontier and it is shown in panel B.

The failure boundaries shown in panel A of Figure 1 are not the end of the story. If organization $j$ fails, then organization $i$ 's value falls discontinuously. In effect, through $i$ 's cross-holding in $j$ and the reduction in $j$ 's value, $i$ bears one-third of $j$ 's failure costs of 50. Organization $i$ then fails if $\frac{2}{3} p_{i}+\frac{1}{3}\left(p_{j}-50\right)<50$. We refer to this new failure threshold as $i$ 's failure frontier conditional on $j$ failing and label it $F F_{i}^{\prime}$. These conditional failure frontiers are shown in panel $\mathrm{C}$.

The conditional failure frontiers identify a region of multiple equilibria due to interdependencies in the values of the organizations. As discussed earlier, this is a different source of multiple equilibria from the familiar bank-run story. The multiple equilibria arise because $i$ 's value decreases discontinuously when $j$ fails and $j$ 's value decreases discontinuously when $i$ fails. It is then consistent for both $i$ and $j$ to survive, in which case the relevant failure frontiers are the unconditional ones, and consistent for both $i$ and $j$ to fail, in which case the relevant failure frontiers are the conditional ones.

\footnotetext{
${ }^{34}$ This is the best-case equilibrium across all possible equilibria; this statement remains true even when we consider multiplicity not arising from interdependencies among organizations.
} 
Panel A. Unconditional failure frontiers

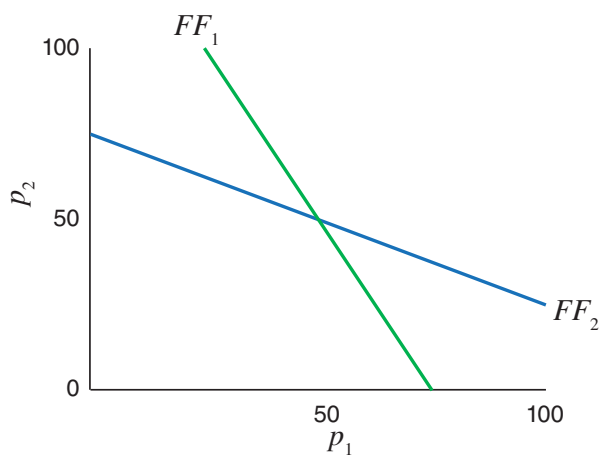

Panel C. Multiple equilibria

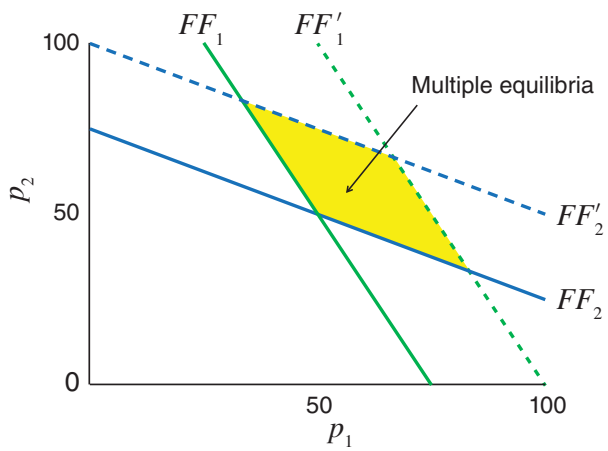

Panel B. The first failure frontier

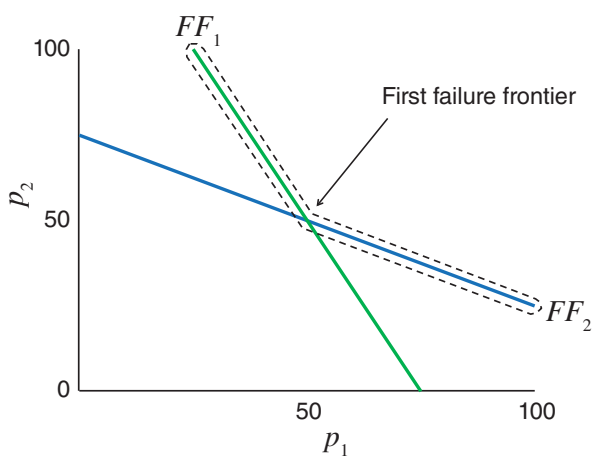

Panel D. Cascades of failure

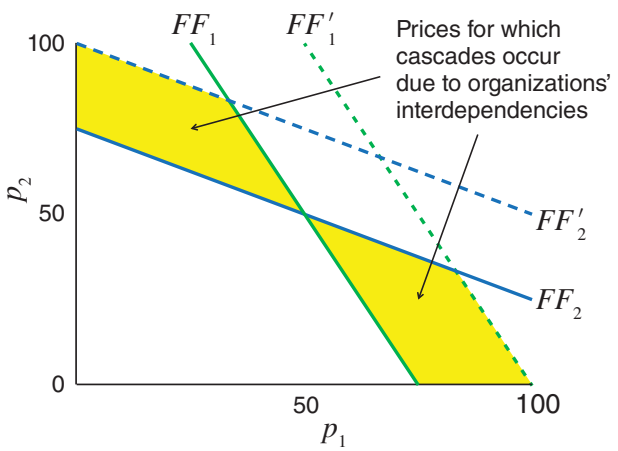

Figure 1. With Positive Cross-Holdings the Discontinuities in Values Generated by the Failure Costs Can Result in Multiple Equilibria and Cascades of Failure

Panel D of Figure 1 identifies the regions where cascades occur in the best-case equilibrium. ${ }^{35}$ When asset prices move from being outside the first failure frontier to being inside this region, the failure of one organization precipitates the failure of the other organization. One organization crosses its unconditional (best-case) failure frontier and the corresponding asset prices are also inside the other organization's conditional failure frontier (which includes the costs arising from the other organization's failure). ${ }^{36}$

A Simple Algorithm for Identifying Cascade Hierarchies.-In simple examples, all the relevant information about exactly who will fail at which asset prices can be represented in diagrams such as those in the previous section. However, the number of conditional failure frontiers grows exponentially with the number of organizations, while adding assets increases the dimensions, making geometric depiction infeasible for larger environments. Thus, while the diagrams provide a useful device

\footnotetext{
${ }^{35}$ Compare with Figure 3 in Gouriéroux, Héam, and Monfort (2012), which makes some of the same points.

${ }^{36}$ As hinted at above, the full set of multiple equilibria is more complex than pictured in Figure 1 and this is discussed in the online Appendix (Sections 7 and 8). For example, the worst-case equilibrium has frontiers further out than those in panel $\mathrm{C}$, as those are based on including failure costs arising from the other organization failing. The worst-case equilibrium is obtained by examining frontiers based on failure costs presuming that both fail, and then finding values consistent with those frontiers. There are also additional equilibria which differ from both the best- and worst-case equilibria - ones that presume one organization's failure but not the other organization's, and find the highest prices consistent with these presumptions.
} 
for introducing ideas, they are of less use practically. In this section, we provide an algorithm which traces the propagation of a specific shock that causes one organization to fail. ${ }^{37}$ As before, we focus on the best-case equilibrium in terms of having the fewest failures and the maximum possible values $v_{i}$.

At step $t$ of the algorithm, let $\mathcal{Z}_{t}$ be the set of failed organizations. Initialize $\mathcal{Z}_{0}=\emptyset$. At step $t \geq 1$ :

(i) Let $\tilde{\mathbf{b}}_{t-1}$ be a vector with element $\tilde{b}_{i}=\beta_{i}$ if $i \in \mathcal{Z}_{t-1}$ and 0 otherwise.

(ii) Let $\mathcal{Z}_{t}$ be the set of all $k$ such that entry $k$ of the following vector is negative:

$$
\mathbf{A}\left[\mathbf{D p}-\tilde{\mathbf{b}}_{t-1}\right]-\underline{\mathbf{v}}
$$

(iii) Terminate if $\mathcal{Z}_{t}=\mathcal{Z}_{t-1}$. Otherwise return to step 1.

When this algorithm terminates at step $T$ (which it will, given the finite number of organizations), the set $\mathcal{Z}_{T}$ corresponds to the set of organizations that fail in the best-case equilibrium. ${ }^{38}$

This algorithm provides us with hierarchies of failures. That is, the various organizations added at each step (the new entries in $\mathcal{Z}_{t}$ compared to $\mathcal{Z}_{t-1}$ ) are organizations whose failures were triggered by the cumulative list of prior failures; they would not have failed if not for that accumulation and, in particular, if not for the failures of those added at the previous step. Thus, $\mathcal{Z}_{1}$ are the first organizations to fail, then $\mathcal{Z}_{2} \backslash \mathcal{Z}_{1}$ are those whose failures are triggered by the first to fail, and so forth.

Note that the sets depend on $\mathbf{p}$ (and $\mathbf{C}$ and $\mathbf{D}$ ), and so each configuration of these can result in a different structure of failures. It is possible to have some $\mathbf{C}$ and $\mathbf{D}$ such that there are some organizations that are never the first to fail, and others who are sometimes the first to fail and sometimes not.

The hierarchical structure of failures has immediate and strong policy implications. If any level of the hierarchy can be made empty, then the cascade stops and no further organization will fail. This suggests that one cost-effective policy for limiting the effect of failures should be to target high levels of the hierarchy that consist of relatively few organizations. ${ }^{39}$ However, such policies may involve more intervention than is necessary. For example, within a wave there could be a single critical organization, the saving of which would prevent any further failure regardless of whether other organizations in the same level failed. Saving an entire level from failure is sufficient for stopping a cascade, but not necessary.

\footnotetext{
${ }^{37}$ This sort of algorithm is the obvious one for finding extreme points of a lattice, and so is standard (for instance, see Theorem 5.1 in Vives 1990). Variations on it appear in the literature on contagions, as in Eisenberg and Noe (2001), Blume et al. (2011), and Rogers and Veraart (2013).

${ }^{38}$ The same algorithm can be used to find the set of organizations that fail in the worst-case equilibrium by instead initializing the set $\mathcal{Z}_{0}$ to contain all organizations and looking for organizations that will not fail, and so forth.

${ }^{39}$ As considered in Section IH.
} 


\section{Defining Integration and Diversification}

One of our contributions is a distinction between the roles of diversification and integration in cascades. Before presenting those results (in the next section), we define the essential distinction between the two network properties.

We say that a financial system becomes more diversified when the number of cross-holders in each organization $i$ weakly increases and the cross-holdings of all original cross-holders of $i$ weakly decrease.

Formally, cross-holdings $\mathbf{C}^{\prime}$ are more diversified than cross-holdings $\mathbf{C}$ if and only if

- $\quad C_{i j}^{\prime} \leq C_{i j}$ for all $i, j$ such that $C_{i j}>0$, with strict inequality for some ordered pair $(i, j)$, and

- $C_{i j}^{\prime}>C_{i j}=0$ for some $i, j$.

Thus, diversification captures the spread in organizations' cross-holdings.

A financial system becomes more integrated if the external shareholders of each organization $i$ have lower holdings, so that the total cross-holdings of each organization by other organizations weakly increase.

Formally, cross-holdings $\mathbf{C}^{\prime}$ are more integrated than cross-holdings $\mathbf{C}$ if and only if $\hat{C}_{i i}^{\prime} \leq \hat{C}_{i i}$ for all $i$, with strict inequality for some $i$. This is equivalent to the condition that

$$
\sum_{j: j \neq i} C_{j i}^{\prime} \geq \sum_{j: j \neq i} C_{j i}
$$

for all $i$, with strict inequality for some $i .{ }^{40}$

Thus, integration captures the depth or extent of organizations' cross-holdings. This can be viewed as an intensive margin. In contrast, diversification pertains to the number of organizations interacting directly with one another, and so is an extensive margin.

It is possible for a change in cross-holdings to increase both diversification and integration. There are changes in cross-holdings that increase diversification but not integration and other changes that increase integration but not diversification.

\section{Essential Ingredients of a Cascade}

To best understand the impact of diversification and integration on cascades, it is useful to identify three ingredients that are necessary for a widespread cascade:

I. A First Failure: Some organization must be susceptible enough to shocks in some assets that it fails.

\footnotetext{
${ }^{40}$ This definition is simple and well suited to our simulations, as in these we will have symmetric values of underlying assets. However, when underlying asset values are asymmetric, there may be changes in cross-holdings consistent with either increasing or decreasing integration that result in substantial changes in the relative values of organizations, and so a more complicated definition is needed. Thus, in our formal results we work with a definition that also holds organizations' market values constant.
} 
II. Contagion: It must be that some other organizations are sufficiently sensitive to the first organization's failure that they also fail. ${ }^{41}$

III. Interconnection: It must be that the network of cross-holdings is sufficiently connected so that the failures can continue to propagate and are not limited to some small component.

Keeping these different ingredients of cascades in mind will help us disentangle the different effects of changes in cross-holdings.

Let us preview some of the ideas, which we soon make precise by imposing some additional structure on the model. As we increase integration (without changing each organization's counterparties), an organization becomes less sensitive to its own investments but more sensitive to other organizations' values, and so first failures can become less likely while contagion can become more likely conditional on a failure. This decreases the circumstances which lead to first failures, making things better with respect to I, while it increases the circumstances where there can be contagion, making things worse with respect to II. Interconnection (III) is not impacted one way or the other, as the network pattern does not change (by assumption). As we increase diversification, organizations become less dependent on any particular neighbor, so contagions can be harder to start, but the network becomes more connected, and so the extent of a contagion broadens (at least up to a point where the network is fully connected). This decreases the circumstances where there can be contagion, making things better with respect to II, while increasing the potential reach of a contagion conditional upon one occurring, making things worse with respect to III.

Understanding this structure makes some things clear. First, integration and diversification affect different ingredients of cascades. Integration affects an organization's exposure to others compared to its exposure to its own assets, while diversification affects how many others one is (directly and indirectly) exposed to. Second, both integration and diversification improve matters with respect to at least one of the cascade ingredients above while causing problems along a different dimension. These trade-offs result in nonmonotonic effects of diversification and integration on cascades, as we now examine in detail.

\section{How Do Cascades Depend on the Diversification and Integration of Cross-Holdings?}

We now turn to our main results.

We begin with some analytic results and then provide additional results via simulations for some random network structures.

\footnotetext{
${ }^{41}$ Note that it need not be an immediate cross-holder that is the sensitive one. Drops in values propagate through the network (as captured by the matrix A), and so the second organization to fail need not be an immediate crossholder, although that would typically be the case.
} 


\section{A. The Consequences of Diversification and Integration: Analytic Results}

A General Result on Integration.-To begin, we prove a general result about how integration affects the extent of cascades. The result permits any initial cross-holdings $\mathbf{C}$, an arbitrary vector of failure costs $\boldsymbol{\beta}$, an arbitrary vector of threshold values $\underline{\mathbf{v}}$, any direct holdings of assets $\mathbf{D}$, and any underlying asset values $\mathbf{p}$.

Recall that the matrices $(\mathbf{C}, \mathbf{D})$ and $\left(\mathbf{C}^{\prime}, \mathbf{D}^{\prime}\right)$ are said to be related by a fair trade at $\mathbf{p}$ if $\mathbf{v}=\mathbf{v}^{\prime}$, where $\mathbf{v}=\mathbf{A p}$ and $\mathbf{v}^{\prime}=\mathbf{A}^{\prime} \mathbf{p}$; the matrix $\mathbf{A}^{\prime}$ is computed as in (5) with $\mathbf{C}^{\prime}$ and $\mathbf{D}^{\prime}$ playing the roles of $\mathbf{C}$ and $\mathbf{D} .^{42}$

PROPOSITION 2: Consider $(\mathbf{C}, \mathbf{D})$ and $\left(\mathbf{C}^{\prime}, \mathbf{D}^{\prime}\right)$ that are related by a fair trade at $\mathbf{p},{ }^{43}$ and such that integration increases: $A_{i j}^{\prime} \geq A_{i j}$ whenever $i \neq j$. There is then the same set of first failures at $(\mathbf{p}, \mathbf{C}, \mathbf{D})$ as at $\left(\mathbf{p}, \mathbf{C}^{\prime}, \mathbf{D}^{\prime}\right)$, and every organization that fails in a cascade at $(\mathbf{p}, \mathbf{C}, \mathbf{D})$ also fails at $\left(\mathbf{p}, \mathbf{C}^{\prime}, \mathbf{D}^{\prime}\right)$.

Proposition 2 states that if we integrate cross-holdings via fair trades, so that organizations end up holding more of each other's investments, then we face more failures in any given cascade that begins. Thus, benefits of integration come only via avoiding first failures. There is a trade-off: integrating can eliminate some first failures. However, given that a first failure occurs, integration only exacerbates the resulting cascade.

The reasoning behind the proposition is as follows. As can be seen immediately from equation (5), when organization $i$ fails and incurs failure costs $\beta_{i}$, it is the $i$ th column of $\mathbf{A}$ which determines who (indirectly) pays these costs. Increasing $A_{i j}$ for all $i$ and $j \neq i$ increases the share of $i$ 's failure costs paid by each of the other organizations. This increases the negative externality $i$ imposes on each organization following its own failure. These other organizations are then more likely to also fail once $i$ fails, and so the number of organizations that fail in the cascade weakly increases.

A Result on Diversification and Integration.-In order to bring diversification into the picture, we specialize the model a bit. Fixing any given level of diversification and integration, a network can typically be rewired to make it more or much less susceptible to cascades of failures. This is an obstruction to obtaining analytical comparative statics with respect to diversification that hold for every network. By working with a random graph model that imposes some structure on the distribution of possible cross-holdings matrices, we can overcome this challenge and make statements that hold with high probability. ${ }^{44}$ The random graph model is tractable yet flexible with respect to degree distributions, making it well suited to the study of diversification. Our analysis of it illustrates some basic intuitions. We then come

\footnotetext{
${ }^{42}$ We show in the online Appendix (Section 3.1) that there are circumstances under which organizations may have incentives to undertake "unfair" trades because of the failure costs.

${ }^{43}$ The definition of a fair trade ignores any failure costs-i.e., the values before and after a trade are calculated as if failures do not occur. This offers a clear benchmark.

${ }^{44}$ When one allows the number of nodes to become arbitrarily large, then various techniques related to laws of large numbers can be applied to deduce connectedness properties of a random network. Thus, one can make statements that hold with high probability when the number of nodes is large. For surveys of techniques relevant to our analysis, see Jackson (2008, chapter 4) and Newman (2010).
} 
back to verify, via simulations, that these intuitions generalize to random networks that are less analytically tractable.

Before introducing any randomness, suppose $\mathbf{G}$ is a fixed matrix with all entries in $\{0,1\}$; we call this an adjacency matrix of an unweighted, directed graph. The interpretation is that $G_{i j}=1$ if organization $i$ has a claim on organization $j$. To make it into a cross-holdings matrix, we posit that a fraction $c$ of each organization is held by other organizations, spread evenly among the $d_{i}=\sum_{j} G_{j i}$ organizations that hold it. We call $d_{i}$ the out-degree of $i$ and analogously define in-degree by $d_{i}^{\text {in }}=\sum_{i} G_{i j}$ to be the number of organizations that $i$ holds. ${ }^{45}$

Thus, for $i \neq j$

$$
C_{i j}= \begin{cases}\frac{c G_{i j}}{d_{j}} & \text { if } d_{j}>0 \\ 0 & \text { otherwise. }\end{cases}
$$

The remaining $1-c$ of the organization is held by its external shareholders, so that $\hat{C}_{j j}=1-c$, if $d_{j}>0$, and 1 otherwise.

Holding $c$ fixed, as the out-degree $d_{j}$ increases, the number of organizations having cross-holdings in $j$ increases, but each of those organizations has lower cross-holdings in $j$. Thus, in this model, increasing $d_{j}$ increases diversification but not integration (assuming that the degree $d_{j}$ starts out being nonzero).

Holding the underlying graph $\mathbf{G}$ fixed, as $c$ increases each organization has lower self-holdings but higher cross-holdings in the other organizations it already holds. Thus, increasing $c$ increases integration but not diversification. This is made precise in the following lemma, which shows how increased integration weakly increases $A_{i j}$ for all $i$ and all $j \neq i$ and strictly increases at least one off-diagonal entry of $\mathbf{A}$ in each column.

LEMMA 2: Suppose that $C_{i j}=c G_{i j} / d_{j}$ for some adjacency matrix $\mathbf{G}$, with $0<c<$ $\frac{1}{2}$ and each $d_{i} \geq 1 .{ }^{46}$ Then $A_{i i}$ is decreasing in $c$ and $A_{i j}$ is increasing in $c$ :

(i) $\frac{\partial A_{i i}}{\partial c}<0$ for each $i$

(ii) $\frac{\partial A_{i j}}{\partial c} \geq 0$ for all $i \neq j$;

(iii) $\frac{\partial A_{i j}}{\partial c}>0$ for all $i \neq j$ such that there is an ownership path ${ }^{47}$ from $i$ to $j$ in $\mathbf{G}$.

\footnotetext{
${ }^{45}$ Note that these terms are intuitive when viewed from the perspective of value flow: out-degree corresponds to how many organizations receive the value that flows out from $i$ by directly holding it. In-degree describes the number of organizations that $i$ holds, and which therefore send value to $i$. In other words, these definitions are made with respect to the network of cascade paths (recall Section IA).

${ }^{46}$ Note that Lemma 2 does not impose any assumptions on the underlying graph $\mathbf{G}$ other than each organization being cross-held by at least one other. Interestingly, the monotonicity identified in Lemma 2 does not always hold for $c>1 / 2$. For such $c$, there are graph structures where further increases in $c$ result in the immediate neighbors of $i$ depending less on $i$. The increase in $A_{i j}$ for nonneighbors of $i$ can come at the expense of both $A_{i i}$ and $A_{i j}$ for $j$ such that $C_{i j}>0$.

${ }^{47}$ Recall footnote 11 and the discussion there.
} 
Next we introduce the random network model. Fix a degree distribution $\pi=\left(\pi_{k, \ell}\right)$, where $\pi_{k \ell}$ is the fraction of nodes that have in-degree $k$ and out-degree $\ell$ and the integer indices satisfy $0 \leq k, \ell \leq n-1$. Let $\mathcal{G}(\pi, n)$ be the set of all directed graphs on $n$ nodes that have degree distribution $\pi$. We say $\pi$ is feasible for $n$ when $\mathcal{G}(\pi, n)$ is nonempty. ${ }^{48}$ A random network with degree distribution $\pi$ is a draw from $\mathcal{G}(\pi, n)$ uniformly at random.

For a given $\pi$, we denote by $\bar{d}=\max \left\{k: \pi_{k \ell}>0\right.$ or $\pi_{\ell k}>0$ for some $\left.\ell\right\}$ the maximum degree of the network and by $\underline{d}=\min \left\{k: \pi_{k \ell}>0\right.$ or $\pi_{\ell k}>0$ for some $\left.\ell\right\}$ the minimum degree. Finally, we define the average directed degree $d$ to be the expected out-degree of the vertex at the end of a link chosen uniformly at random from $\mathcal{G}(\boldsymbol{\pi}, n) \cdot{ }^{49}$ This is a basic measure of average diversification in the graph that overweights organizations held by many others, and turns out to be the right one for our purposes. Together, the three parameters $\underline{d}, \bar{d}$, and $d$ operationalize the notion of diversification in this random network model; we take the average degree $d$ to be positive.

Each organization has a single asset of value 1 ( so $\mathbf{D}=\mathbf{I}$ and $\mathbf{p}=(1, \ldots, 1)$ ). We set all organizations' thresholds $\underline{v}_{i}$ to a common $\underline{v} \in(0,1)$, and set $\beta_{i}=p_{i}$, so that a failing organization has its proprietary asset completely devalued.

Define $\tilde{v}_{\min }=\frac{1-c}{1-c \underline{d} / \bar{d}}$ and $\tilde{v}_{\max }=\frac{1-c}{1-c \bar{d} / \max \{\underline{d}, 1\}} \cdot 50$

How does the degree distribution, $\pi$, affect the extent of cascades? Let $\mathbf{G}$ be a random draw of a network with $n$ nodes and degree distribution $\pi$. Let $f(\pi, n)$ be the expected fraction of organizations that fail if the network is given by $\mathbf{G}$ and one proprietary asset value $p_{i}$ is devalued to 0 , with $i$ selected uniformly at random.

PROPOSITION 3: If one proprietary asset fails (uniformly at random), a nonvanishing fraction of organizations fail if and only if there are intermediate levels of both integration and diversification.

In particular, consider a degree distribution $\pi$ with associated average directed degree $d$, maximum degree $\bar{d}$, and minimum degree $\underline{d}$; and let $\left(n_{k}\right)$ be an infinite sequence of natural numbers such that $\pi$ is feasible for each $n_{k}$.

3A. The fraction of failures tends to $0\left(f\left(\pi, n_{k}\right) \rightarrow 0\right)$ if either of the following conditions holds:

(i) $d<1$ (diversification is too low), or

(ii) $\underline{d}>\frac{c(1-c)}{\tilde{v}_{\min }-\underline{v}}$ (diversification is too high, or integration is too high or low).

\footnotetext{
${ }^{48}$ For $\mathcal{G}(\pi, n)$ to be a nonempty set, some basic relations have to be satisfied by $\pi$ : (i) $n \pi_{i j}$ is always a (nonnegative) integer, since it must be a number of nodes; (ii) $\sum_{k \ell} k \pi_{k \ell}=\sum_{k \ell} \ell \pi_{k \ell}$, since each is equal to the number of directed edges in the graph divided by $n$.

${ }^{49}$ This depends only on $\pi$. To see this, let $\phi_{\ell}$ be the probability that a node of out-degree $\ell$ is found by following a randomly chosen edge; we can see that $\phi_{\ell}=\sum_{k} k \pi_{k \ell} / \sum_{\ell, k} k \pi_{k \ell}$. Now note that $d=\sum \ell \phi_{\ell}$.

${ }^{50}$ These serve as lower and upper bounds, respectively, on organization values, as verified in the proof of Proposition 3; we take $c<\max \{\underline{d}, 1\} / \bar{d}$, so that these are well defined.
} 
3B. The fraction of failures is nonvanishing $\left(\lim _{\inf _{k}} f\left(\pi, n_{k}\right)>0\right)$ if both of the following conditions hold:

(i) $d>1$ (diversification is not too low), and

(ii) $\bar{d}<\frac{c(1-c)}{\tilde{v}_{\max }-\underline{v}} \quad$ (diversification is not too high and integration is intermediate).

Proposition 3 documents a nonmonotonicity of failures in diversification and integration. Proposition $3 \mathrm{~A}$ shows that if either integration or diversification is extreme (low or high), then there can be no substantial contagion: $3 \mathrm{~A}(\mathrm{i})$ is satisfied if diversi-

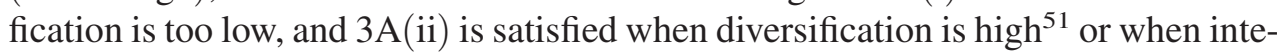
gration is high or low ( $c$ is close to 0 or 1 ). In other words, contagion can occur only if both integration and diversification are intermediate. Proposition $3 \mathrm{~B}$ then gives a sufficient condition: upper and lower bounds on the diversification parameters $\bar{d}$ and $d$, respectively, ${ }^{52}$ specifying the intermediate range in which contagion occurs. ${ }^{53}$

The intuition for Proposition 3 is as follows. If $c$ is very low, then no firm holds enough of its counterparties for contagion to propagate. If $c$ is very high, then no firm is sufficiently exposed to its own asset for a first failure to happen. So consider the range where $c$ is intermediate. For random graphs of the type we study here, once the average directed degree $d$ crosses the threshold 1, the graph structure changes from many isolated components of vanishing size to a giant component of nonvanishing size. It starts out small, but increases in size as $d$ grows. Thus, if $d<1$, contagion to a positive fraction of organizations following the failure of a single proprietary asset is impossible. At the other extreme, once $\underline{d}>\frac{c(1-c)}{\tilde{v}_{\min }-\underline{v}}$, a single organization's failure will not cause a sufficient decrease in the value of any other organization to induce a second failure. When integration and diversification are intermediate, so that none of these obstructions to contagion occur, part B of the proposition states that a (nonvanishing) fraction of organizations fail.

The reasoning above makes use of properties of large networks. Regardless of the parameter values, when there are only a small number of organizations, networks with intermediate connectedness are realized with nontrivial probability. Thus, in settings with very few critical organizations, one has to rely on direct calculations (e.g., see the core-periphery analysis in Section IVA).

\section{B. The Different Roles of Diversification and Integration: Simulations on Random Networks}

We now show that the analytic results of the previous section hold in other classes of simulated random networks. We also derive some richer insights into comparative statics in various levels of diversification and integration.

\footnotetext{
${ }^{51}$ Note that as $c(1-c) \leq 1 / 4$ for all $c \in(0,1), 3 \mathrm{~A}(\mathrm{ii})$ is always satisfied for all $\underline{d}>1 /\left(4\left(\tilde{v}_{\min }-\underline{v}\right)\right)$.

${ }^{52}$ Fixing a ratio $\bar{d} / \underline{d}<1 / c$, the right-hand side of $3 \mathrm{~B}$ (ii) is constant in $\bar{d}$; in this sense $3 \mathrm{~B}(\mathrm{ii})$ is a true upper bound on $\bar{d}$.

${ }^{53}$ Observe that when the graph is regular, so that $\bar{d}=d=\underline{d}$, then $\tilde{v}_{\max }$ and $\tilde{v}_{\min }$ become identical and the result becomes fully tight, with no distance between the necessary and the sufficient condition for contagion.
} 
Simulated Random Networks.-To illustrate how increased diversification and increased integration affect the number of organizations that fail in a cascade following the failure of a single organization's assets, we specialize the model.

Each organization has exactly one proprietary asset, so that $m=n$ and $\mathbf{D}=\mathbf{I}$. This keeps the analysis uncluttered, and allows us to focus on the network of cross-holdings.

For simplicity, we also start with asset values of $p_{i}=1$ for all organizations, and have common failure thresholds $\underline{v}_{i}=\theta v_{i}$, for a parameter $\theta \in(0,1)$, where $v_{i}$ is the starting value of organization $i$ when all assets are at value 1 . In case an organization fails, it loses its full value, so that $\beta_{i}=\underline{v}_{i}$.

The cross-holdings are derived from an adjacency matrix $\mathbf{G}$ with entries in $\{0,1\}$, where $G_{i j}=1$ indicates that $i$ has cross-holdings in $j$ and we set $G_{i i}=0$.

Again, a fraction $c$ of each organization is held by other organizations, spread evenly among the $d_{i}=\sum_{j} G_{j i}$ organizations that hold it as in Section IIIA. The remaining $1-c$ of the organization is held by its external shareholders, so that $\hat{C}_{i i}=1-c$. The same adjustments are made for degree- 0 nodes as before.

To illustrate the effects of increasing diversification and increasing integration on cascades we examine a setting where connections between organizations are formed at random, with each organization having cross-holdings in a random set of other organizations.

In particular, we form a directed random graph, with each directed link having probability $d /(n-1)$, so that the expected in-degree and out-degree of any node is $d$. More precisely, the adjacency matrix of the graph is a matrix $\mathbf{G}$ (usually not symmetric), where $G_{i j}$ for $i \neq j$ are i.i.d. Bernoulli random variables each taking value 1 with probability $d /(n-1)$ and 0 otherwise.

To examine the effects of increasing diversification (increasing $d$ ) and increasing integration (increasing $c$ ), we simulate an organization's proprietary asset failing and record the number of organizations that fail in the resulting cascade.

We follow a simple algorithm:

Step 1: Generate a directed random network $\mathbf{G}$ with parameter $d$ as described above.

Step 2: Calculate the matrix $\mathbf{C}$ from $\mathbf{G}$ as described in Section IIIA.

Step 3: All organizations start with asset values of $p_{i}=1$. Calculate organizations' initial values $v_{i}$ and set $\underline{v}_{i}=\theta v_{i}$ for some $\theta \in(0,1)$.

Step 4: Pick an organization $i$ uniformly at random and drop the value $\left(p_{i}\right)$ of $i$ 's proprietary asset to 0.54

Step 5: Assuming all other asset values $\left(p_{j}\right.$ for $\left.j \neq i\right)$ stay at 1 , calculate the best-case equilibrium using the algorithm from Section IIB.

\footnotetext{
${ }^{54}$ Thus, we are focusing on a case where an organization's proprietary project is shut down upon failure. While clearly not the only case of interest, it is a common outcome in practice.
} 
Panel A. Effects of diversification: the percentage of organizations failing as a function of expected degree for $\theta=0.93(c=0.5, n=100)$

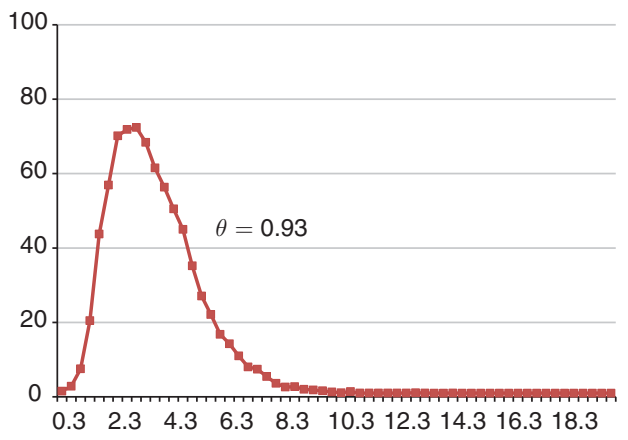

Panel B. Effects of diversification for several failure thresholds: percentage of organizations failing as a function of expected degree for various levels of $\theta(c=0.5, n=100)$

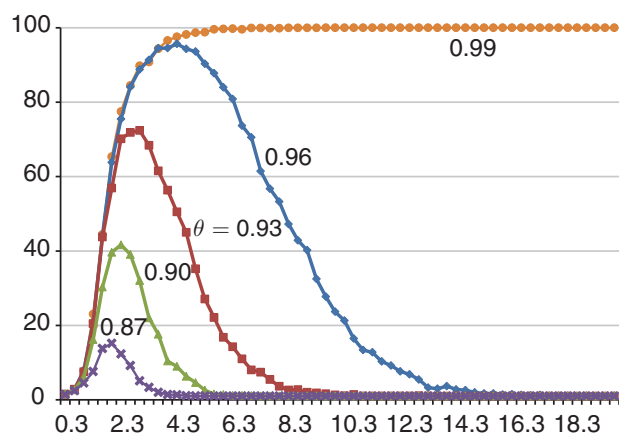

Figure 2. How Diversification (the Average Number of Other Organizations that an Organization Cross-Holds) Affects the Percentage of Organizations Failing (Averaged over 1,000 simulations)

Note: The $x$-axis corresponds to diversification in terms of the expected degree in the random network of cross-holdings.

The main outcome variable we track is the number of failures in the best-case equilibrium.

The Consequences of Diversification: It Gets Worse before It Gets Better-For our simulations, we consider $n=100$ nodes and work with a grid on expected degree $d$ between 1 and 20 (varying in increments of $1 / 3$ ). We work with values of $\theta \in[0.8,1]$.

Our first exercise is to vary the level of diversification (the expected degree $d$ in the network) while holding other variables fixed and to see how the number of organizations (out of 100) which fail varies with the diversification.

Panels A and B of Figure 2 illustrate how the proportion of organizations which fail changes as the level of diversification $(d)$ is varied (fixing integration at $c=0.5$ ).

Panel A shows the result for a level of the failure threshold $(\theta=0.93)$ for which the curves display their typical nonmonotonicities clearly. When $d$ is sufficiently low (1.5 or below), then we see the percentage of organizations that fail is less than 20. At that level, the network is not connected; a typical organization has direct or indirect connections through cross-holdings to only a small fraction of others, and any contagion is typically limited to a small component. As $d$ increases (in the range of two to six other organizations) then we see substantial cascades affecting large percentages of the organizations. In this middle range, the network of cross-holdings has two crucial properties: it is usually connected, ${ }^{55}$ and organizations still have large enough cross-holdings in individual other organizations so that contagion can occur. This is the "sweet spot" where ingredients II (contagion) and III (interconnection) are present and strong-contagion is possible and there is enough interconnection for a cascade to spread. As we continue to increase diversification, the 
Panel A. Low diversification

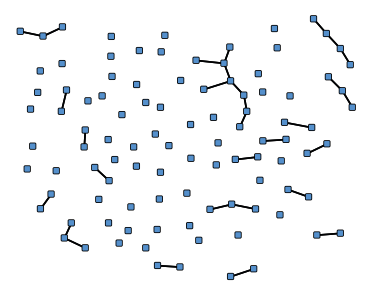

Panel B. Medium diversification

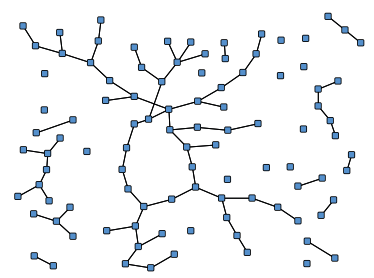

Panel C. High diversification

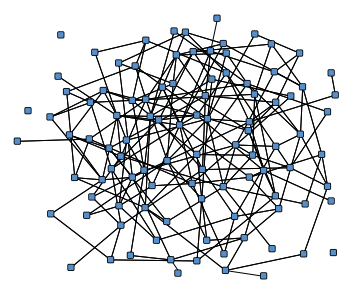

Figure 3. Example Random Networks (Plotted here with undirected edges) FOR DIFFERENT LEVELS OF DIVERSIFICATION

Note: The diagrams demonstrate the transition from panel A (many disconnected components) to panel B (a large component where each node has few neighbors) to panel $\mathrm{C}$ (a large component in which each node has many neighbors).

extent of cascades falls, as diversification is now lowering the chance that contagion occurs. In summary, there is constantly a trade-off between II and III, but initially III dominates as diversification leads to dramatic changes in the connectedness of the network. Then II dominates: once the network is connected, the main limiting force is the extent to which the failure of one organization sparks failures in others, which is decreasing with diversification. These three regimes are illustrated in Figure 3

Panel B of Figure 2 shows how these effects vary with $\theta$. Higher values of $\theta$ correspond to higher failure thresholds, and so it becomes easier to trigger contagions. This leads to increases in the curves for all levels of diversification. Essentially, increasing $\theta$ leads to a more fragile economy across the board.

The main results in Section IIIA provide analytical support for the nonmonotonicity due to diversification identified in the simulations and help identify the forces behind the nonmonotonicity. With low levels of diversification, contagions are difficult to start and will frequently die out before affecting many organizations. Ingredient III is not present, as the network of cross-holdings is not connected. Even if all organizations directly or indirectly dependent on the failing organization $i$ (those $j$ such that $A_{i j}>0$ ) also fail in the cascade, there are sufficiently few such organizations that the cascade dies out quickly and is small. As we increase diversification into intermediate levels, we see an increase in the number of organizations that fail in a cascade. Since network components are larger, the failure of any one organization infects more other organizations, and more organizations are drawn into the cascade. However, as we continue to diversify cross-holdings, eventually the increased diversification leads to a decrease in exposure of any one organization to any other, and so ingredient II is not present as no organization depends very much on any other.

Cascades Are Larger but Less Frequent in More Integrated Systems.-Next, we consider the implications of increased integration in our simple model on the depth of cascades, as illustrated in Figure 4.

Panels A and B of Figure 4 illustrate how the proportion of organizations which fail changes as the level of integration is varied from $c=0.1$ to 0.5 , for two different values of $\theta$ (the fraction of initial value that must be retained for an organization to avoid failure). As integration is increased, the curves all shift upward and we see increased cascades. 
Panel A. Five levels of integration and the percentage of organizations failing as a function of expected degree $(\theta=0.93),(n=100)$

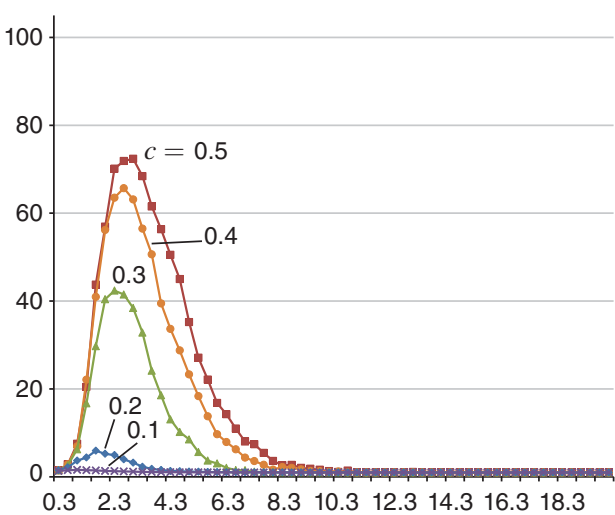

Panel B. Five levels of integration and the percentage of organizations failing as a function of expected degree $(\theta=0.96),(n=100)$

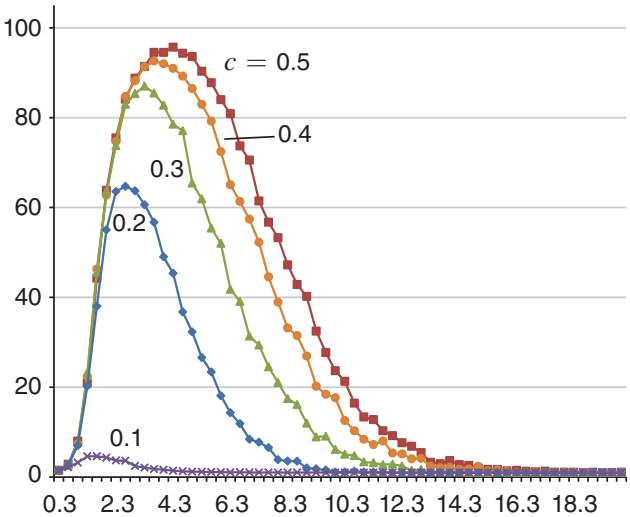

Figure 4. How Integration (the Fraction $c$ of a Typical Portfolio Held by Other Organizations) Affects the Percentage of Organizations Failing (Averaged over 1,000 simulations)

Notes: The $x$-axis corresponds to the diversification level (the expected degree in the random network of cross-holdings). The two figures work with different failure thresholds and depict how the size of cascades varies with the level of integration $c$ ranging from 0.1 to 0.5 .

Although the effects in panels A and B show unambiguous increases in cascades as integration increases, they work with levels of $c \leq 0.5$, for which there is not much of a trade-off. In particular, for $c \leq 0.5$ the initial organization whose asset price is dropped to 0 always fails (in the range of $\theta \geq 0.8$ considered in the simulations). As $c$ is increased beyond 0.5 , eventually the integration level begins to help avoid first failures, because each organization is less exposed to the failure of its own proprietary asset. Then we see the trade-off between ingredients I (first failure) and II (contagion) that is present as integration is varied (holding diversification constant, so III-having to do with the global connectedness of the network-is not affected). We can see this in Figure 5.

Figure 5 shows that as integration increases to very high levels, the percentage of first failures declines: organizations are so integrated that the drop in the value of an organization's own investments is less consequential to it, and so there is no first failure.

To summarize, increasing integration (as long as it is not already very high) makes shocks more likely to propagate to neighbors in the financial network and increases contagion via the mechanism of II. For very high levels of integration, each organization begins to carry something close to the market portfolio, and so any first failure caused by the devaluation of a single proprietary asset becomes less likely.

\section{Alternative Network Structures}

Additional insights emerge from examining some other random graph models of financial interdependencies. 


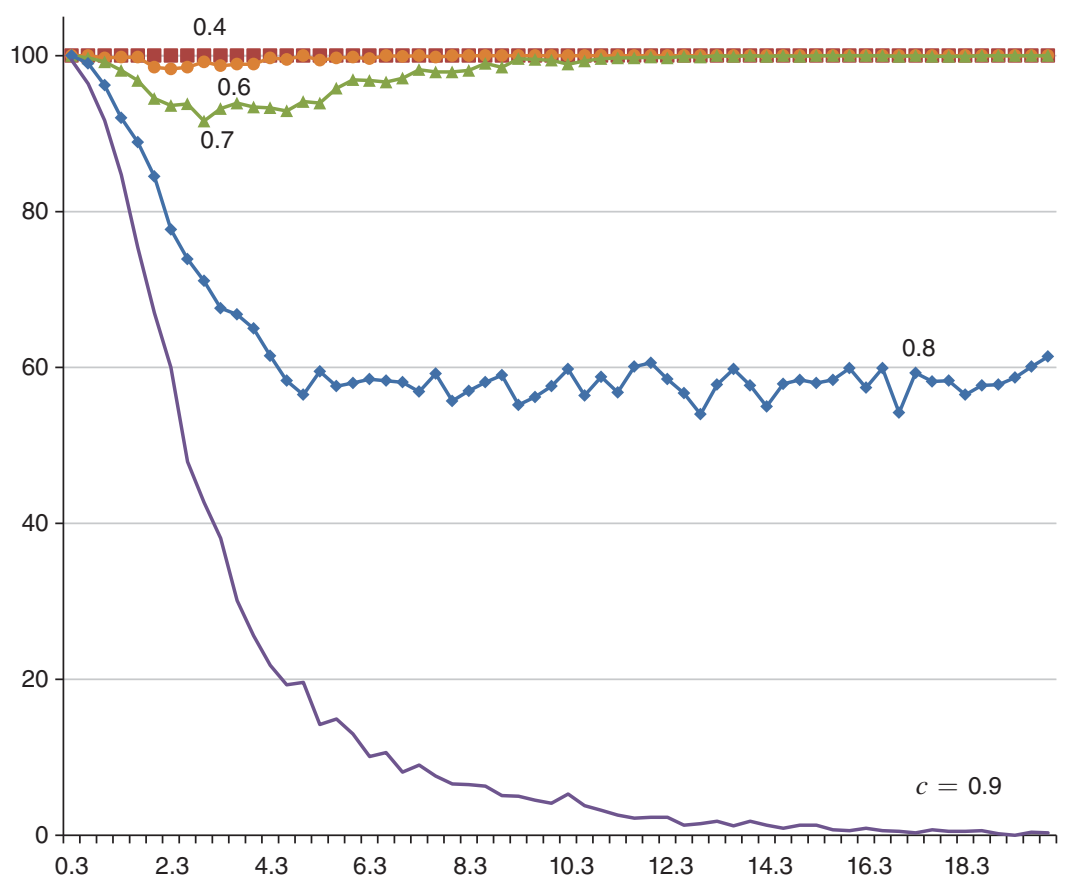

Figure 5. How Integration Affects the Percentage of "First Failures"

Notes: The percentage of simulations with at least one organization failing, for various levels of integration $c$ from 0.4 to 0.9 , with the $x$-axis tracking diversification (expected degree) in the network. The failure threshold is constant at $\theta=0.8$.

\section{A. A Core-Periphery Model}

As a stylized representation of the interbank lending market, we examine a core-periphery model where 10 large organizations are completely connected among themselves, and each of 90 smaller organizations has one connection to a random core organization. ${ }^{56}$ Each of the 10 large core organizations has proprietary assets with an initial value of 8 . Each of the 90 peripheral organizations has proprietary assets with an initial value of 1 .

We then vary different facets of integration: ${ }^{57}$ the level $C_{C C}$ of cross-holdings of each core organization by other core organizations, the level $C_{P C}$ of cross-holdings of each core organization by peripheral organizations, and the level $C_{C P}$ of cross-holdings of each peripheral organization by core organizations. The remaining private holdings, $\hat{C}_{i i}$, are as follows: $\hat{C}_{i i}=1-C_{C C}-C_{P C}$ for a core organization, and $\hat{C}_{i i}=1-C_{C P}$ for a peripheral one.

We first explore what happens when a core organization fails. As we see in the left-hand part of panel A of Figure 6, the fraction of peripheral organizations which

\footnotetext{
${ }^{56}$ Soramäki et al. (2007) map the US interbank network based on the Fedpayments system. They identify a clique of 25 completely connected banks (including the very largest ones), and thousands of less connected peripheral regional and local banks.

${ }^{57}$ Note that in this model the diversification (degree) structure is essentially fixed given the structure of ten completely interconnected organizations and the peripheral ones each having one connection; the only randomness comes from the random attachment of each peripheral organization to a single core organization.
} 
Panel A. One core organization's asset initially fails

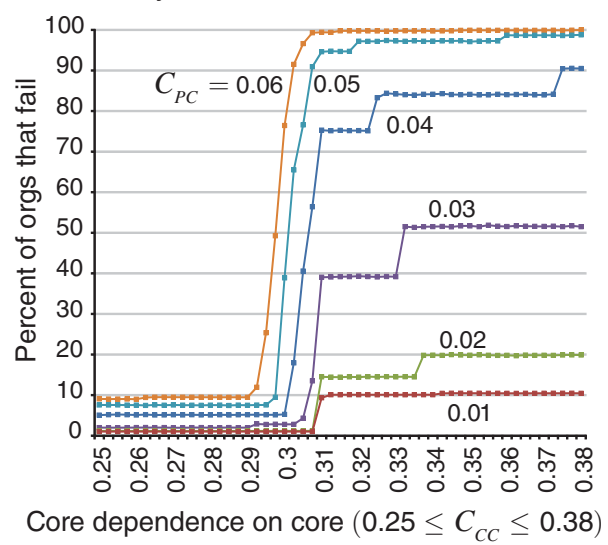

Panel B. One peripheral organization's asset initially fails

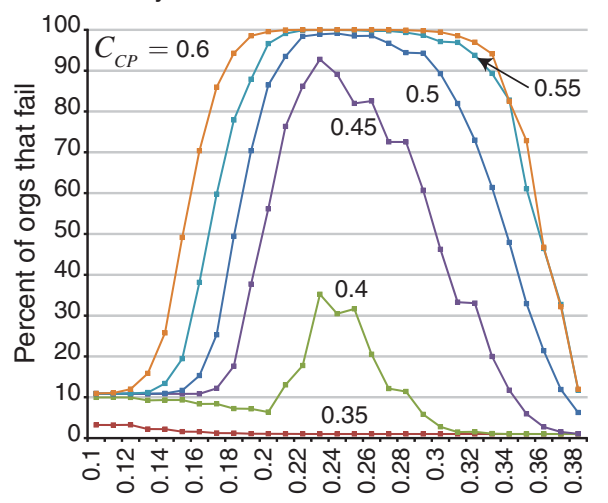

Core dependence on core $\left(0.1 \leq C_{C C} \leq 0.38\right)$

Figure 6. The Consequences of Failure in the Core-Periphery Model

Notes: The $x$-axis is the fraction of each core organization cross-held by other core organizations (integration of core to core). In panel A, curves correspond to different levels of cross-holdings of each core organization by peripheral organizations. In panel $\mathrm{B}$, they correspond to different levels of cross-holdings of peripheral organizations by core ones. The failure threshold is $\theta=0.98$.

fail along with the core organization is increasing in $C_{P C}$. Once the core organizations become sufficiently integrated among themselves, starting around $C_{C C}=0.29$, the core organization's failure begins to cascade to other core organizations, and then wider contagion occurs. How far this ultimately spreads is governed by the combination of integration levels.

The more subtle effects are seen in panel B. The curves are layered in terms of integration between the core and periphery $C_{C P}$, with increased integration leading to higher failure rates due to an initial failure of a peripheral organization. However, the magnitude of the failure rates is initially increasing in core integration $\left(C_{C C}<0.25\right)$ and then decreasing in core integration $\left(C_{C C}>0.25\right)$. Initial increases in core integration enable contagion from one core organization to another, which leads to widespread cascades. Once core integration becomes high enough, however, core organizations become less exposed to their own peripheral organizations, and so then are less prone to fail because of the failure of a peripheral organization.

\section{B. A Model with Segregation among Sectors}

Second, we consider a model which admits segregation (homophily) among different segments of an economy-for instance among different countries, industries, or sectors. In this model, there are ten different groups of ten nodes each. The key feature being varied is the relative intensity of nodes' connections with others in their own group compared to other groups. This captures the difference between integration across industries and integration within industries. Varying this difference leads to the results captured in Figure 7. An obvious effect is that increasing homophily can eventually sever connections between groups of organizations and lead to lower contagion. However, as we see in Figure 7, the curves associated with different levels of diversification (expected degrees $d$ ) cross each other. With 


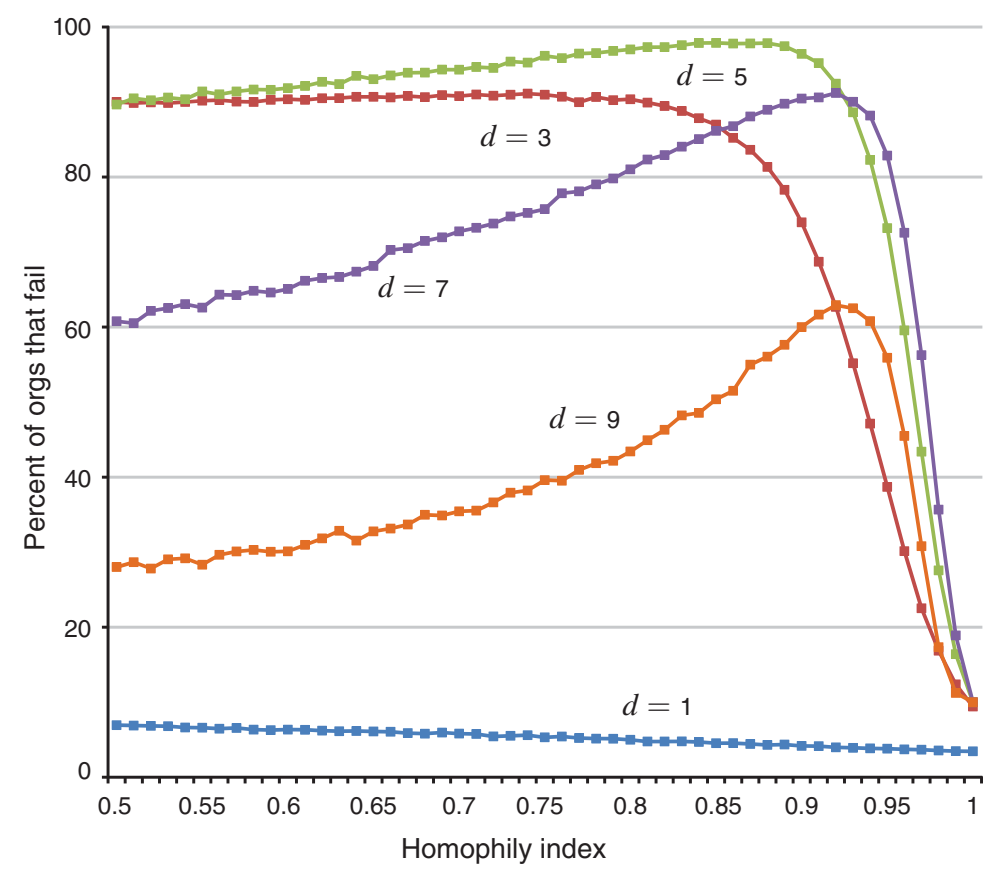

Figure 7. Ten Groups of Ten Organizations Each

Notes: The $y$-axis is the fraction of organizations that fail as a function of the homophily. The $x$-axis is the fraction of expected cross-holdings in same-type organizations. Curves correspond to different diversification levels (expected degrees $d$ ). The failure threshold is $\theta=0.96$.

medium diversification (e.g., $d=3$ or $d=5$ ) there is initially a higher level of contagion than with higher diversification (e.g., $d=7$ or $d=9$ ). This is because organizations are more susceptible to each other with medium degrees than with high degrees and the network is still connected enough to permit widespread contagion. However, lower-degree networks fragment at lower levels of homophily than high-degree networks. So at high levels of homophily, lower-degree networks are actually more robust. For example, once at least 95 percent of relationships are within an organization's own group (in expectation), then we see lower contagion rates with a diversification level $d=5$ than with $d=7$.

\section{Power Law Distributions}

We also examined networks with more extreme degree distributions, such as a power-law distribution. Those results are described in detail in Section 4.1 in the online Appendix and are in line with the original regular networks. More extreme exponents in the power law actually lead to smaller contagions on average, but also lead to larger contagions conditional on some high-degree organization's failure.

\section{Correlated and Common Assets}

An important concern that emerged from the recent financial crisis is that many organizations may have investments with correlated payoffs, which could potentially 
exacerbate contagions, as many organizations' values may be low at the same time. In Sections 4.2 and 4.3 of the online Appendix we examine two variations with correlated values. As one might expect, increasing correlation increases the failure rate. The more interesting part is that the increase occurs abruptly at a particular level of correlation.

We also examine a model in which organizations have some holdings of both an idiosyncratic and a common asset, with the possibility of leverage in holdings of the common asset. Some organizations are long the asset and others can be short. This results in some interesting patterns in cascades: even low leverage levels can lead to increased cascades by increasing organizations' exposures. However, organizations that are short the common asset might escape a cascade triggered by a shock to that asset.

\section{Illustration with European Debt Cross-Holdings}

We close the paper with an illustration of the model with data on the crossholdings of debt among six European countries (France, Germany, Greece, Italy, Portugal, and Spain). We include this as a proof of concept, and emphasize that the crude estimates which we use for cross-holdings make this noisy enough that we do not see the conclusions as robust, but merely as illustrative of the methodology. 58

We take the fundamental asset owned by each country to be its fiscal stream; by exchanging cross-holdings, countries acquire holdings whose value depends on the value of others' fiscal streams as well as on their own. We model failure as being triggered by a certain percentage loss in the value of a country's aggregate holdings. In the simulations, when a country "fails," it defaults on 50 percent of its obligations to foreign countries - an arbitrary choice, but not unfounded, as we see from the write-down of Greek debt. Such losses may arise for various reasons: discontinuous changes in government policies of how to make use of fiscal streams; government decisions not to honor obligations (at which point it makes sense to do so discontinuously); discontinuities in the fiscal streams themselves (due to strikes, discontinuous changes in foreign investments, bank runs, and so forth). Indeed, all of these phenomena were observed in the recent Greek crisis. Finally, for the purpose of this illustrative exercise, we treat these countries as a closed system with no holdings by other countries outside of these six.

\section{A. The Data}

Data on the cross-holdings are for the end of December 2011 from the BIS (Bank for International Settlements) Quarterly Review (Table 9B). The data used for this exercise are the consolidated foreign claims of banks from one country on debt obligations of another country. The data looks at the immediate borrower rather than the

\footnotetext{
${ }^{58}$ See Upper (2011) for a nice review of the empirical literature simulating the effects of shocks to financial systems. Explicit losses due to bankruptcy are not usually considered in this literature, but an important exception is Elsinger, Lehar, and Summer (2006), who find that these costs can make a large difference to the extent of contagion in simulation analysis. Our approach is well-suited to developing a deeper analysis of the propagation of discontinuities, as we examine the various levels of a cascade - which failures cause which others. This is illustrated in this section.
} 
final borrower ${ }^{59}$ when a bank from a country different from the final borrower serves as an intermediary. ${ }^{60}$

This gives the following raw cross-holdings matrix, where the column represents the country whose debt is being held and the row is the country which holds that debt. So, for example, through their banking sectors Italy owes France $\$ 329,550 \mathrm{M}$, while France owes Italy only \$40,311M.

$\left(\begin{array}{lcccccc} & (\text { France }) & (\text { Germany }) & (\text { Greece }) & (\text { Italy }) & \text { (Portugal) } & \text { (Spain) } \\ \text { (France }) & 0 & 198,304 & 39,458 & 329,550 & 21,817 & 115,162 \\ \text { (Germany) } & 174,862 & 0 & 32,977 & 133,954 & 30,208 & 146,096 \\ (\text { Greece }) & 1,960 & 2,663 & 0 & 444 & 51 & 292 \\ (\text { Italy }) & 40,311 & 227,813 & 2,302 & 0 & 3,188 & 26,939 \\ \text { (Portugal) } & 6,679 & 2,271 & 8,077 & 2,108 & 0 & 21,620 \\ (\text { Spain }) & 27,015 & 54,178 & 1,001 & 29,938 & 78,005 & 0\end{array}\right)$

To convert the above matrix into our fractional cross-holdings matrix, $\mathbf{C}$, we then estimate the total amount of debt issued by each country. To do this, we estimate the ratio of total debt held outside the issuing country by $1 / 3$, in line with estimates by Reinhart and Rogoff (2011). Then, the formula $\mathbf{A}=\hat{\mathbf{C}}(\mathbf{I}-\mathbf{C})^{-1}$ implies that A is:

$\left(\begin{array}{lcccccc} & (\text { France }) & \text { (Germany) } & (\text { Greece }) & (\text { Italy }) & \text { (Portugal) } & \text { (Spain) } \\ (\text { France }) & 0.71 & 0.13 & 0.13 & 0.17 & 0.07 & 0.11 \\ (\text { Germany }) & 0.18 & 0.72 & 0.12 & 0.11 & 0.09 & 0.14 \\ (\text { Greece }) & 0.00 & 0.00 & 0.67 & 0.00 & 0.00 & 0.00 \\ (\text { Italy }) & 0.07 & 0.12 & 0.03 & 0.70 & 0.03 & 0.05 \\ \text { (Portugal) } & 0.01 & 0.00 & 0.02 & 0.00 & 0.67 & 0.02 \\ (\text { Spain }) & 0.03 & 0.03 & 0.02 & 0.02 & 0.14 & 0.68\end{array}\right)$

The matrix A can be pictured as a weighted directed graph, as in Figure 8. The arrows show the way in which decreases in value flow from country to country. For example, the arrow from Greece to France represents the value of France's claims on Greek assets, and thus how much France is harmed when Greek debt loses value. (Thus, in the terminology of Section IA, paths in this network correspond to cascade paths.) The areas of the ovals represent the value of each country's direct holdings

\footnotetext{
${ }^{59}$ Which basis is appropriate is discussed in Section 10 of the online Appendix.

${ }^{60}$ For illustrative purposes, we examine holdings at a country level, so that all holdings of Italian debt by banks or other investors in France are treated as being held by the entity "France," and we suppose that substantial losses by banks and investors in France would lead to a French default on national debt. It would be more accurate to disaggregate and build a network of all organizations and investors, if such data were available.
} 


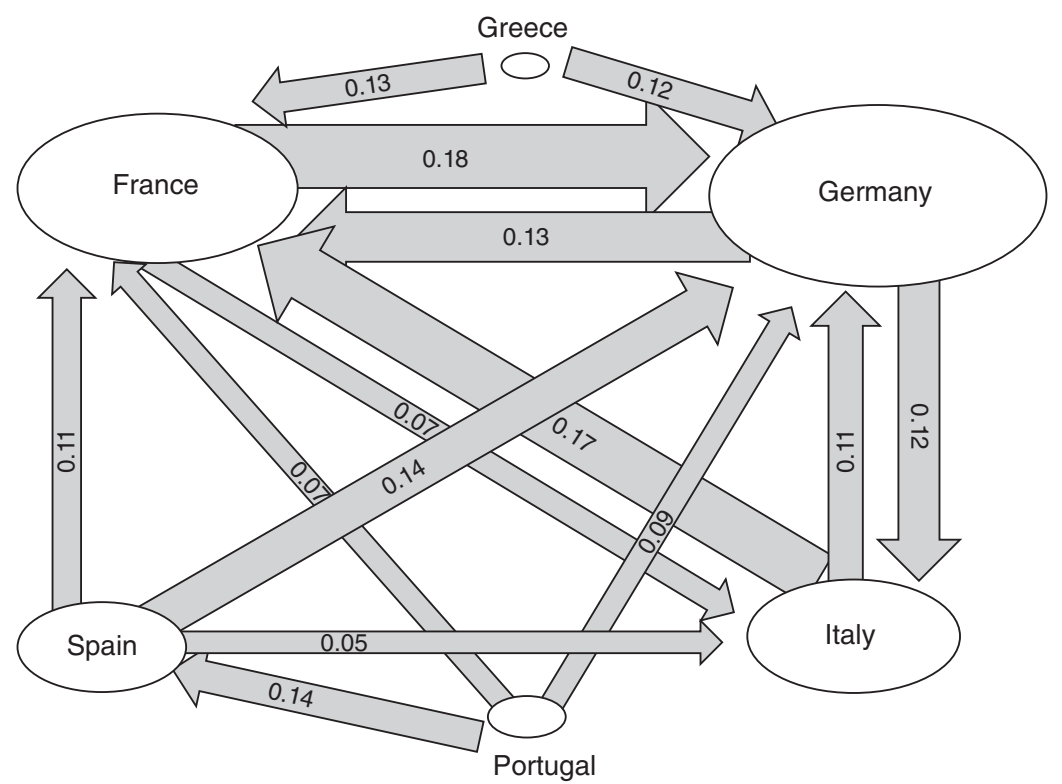

Figure 8. INTERDEPENDENCIES IN EUROPE

Notes: The matrix A, describing how much each country ultimately depends on the value of others' debt. The widths of the arrows are proportional to the sizes of the dependencies, with dependencies less than 5 percent excluded; the area of the oval for each country is proportional to its underlying asset values.

of primitive assets. All dependencies of less than 5 percent have been excluded from Figure 8 (but appear in the table above).

We treat the investments in primitive assets as if each country holds its own fiscal stream, which is used to pay for the debt, and presume that the values of these fiscal streams are proportional to GDP (gross domestic product). Thus, $\mathbf{D}=\mathbf{I}$ and $\mathbf{p}$ is proportional to the vector of countries' GDPs. ${ }^{61}$ Normalizing Portugal's 2011 GDP to 1 , the initial values in 2011 are $\mathbf{v}_{0}=\mathbf{A p}$,

$\left(\begin{array}{llllll}0.71 & 0.13 & 0.13 & 0.17 & 0.07 & 0.11 \\ 0.18 & 0.72 & 0.12 & 0.11 & 0.09 & 0.14 \\ 0.00 & 0.00 & 0.67 & 0.00 & 0.00 & 0.00 \\ 0.07 & 0.12 & 0.03 & 0.70 & 0.03 & 0.05 \\ 0.01 & 0.00 & 0.02 & 0.00 & 0.67 & 0.02 \\ 0.03 & 0.03 & 0.02 & 0.02 & 0.14 & 0.68\end{array}\right) \cdot\left(\begin{array}{c}11.6 \\ 14.9 \\ 1.3 \\ 9.2 \\ 1.0 \\ 6.3\end{array}\right)=\left(\begin{array}{l}12.7 \text { (France) } \\ 14.9 \text { (Germany) } \\ 0.8 \text { (Greece) } \\ 9.4 \text { (Italy) } \\ 0.9 \text { (Portugal) } \\ 5.4 \text { (Spain) }\end{array}\right)$

\footnotetext{
${ }^{61}$ We work in the scale of GDPs - that is, we do not carry around an explicit constant of proportionality relating the value of the fiscal streams $\mathbf{p}$ to the value of GDP; we simply take the entries of the vector $\mathbf{p}$ to be the GDP values.
} 


\section{B. Cascades}

To illustrate the methodology, we consider a simple scenario. The failure thresholds $\underline{v}_{i}$ are set to $\theta$ multiplied by 2008 values. ${ }^{62}$ If a country fails, then the loss in value is $\underline{v}_{i} / 2$, so that half the value of its debt is lost.

We examine the best equilibrium values for various levels of $\theta$. Greece's value has already fallen by well more than 10 percent, and so it has hit its failure point for all of the values of $\theta$ that we look at. We vary $\theta$ and see which cascades occur. Table 1 records the results of these simulations.

We see that Portugal is the first failure to be triggered by a contagion. Although it is not particularly exposed to Greek debt directly, the fact that its GDP has dropped substantially means that it is triggered once we get to $\theta=0.935$. Once Portugal fails, then Spain fails due to its poor initial value and its exposure to Portugal. Then the large size of Spain, and the exposure of France and Germany to Spain, cause them to fail. Pushing $\theta$ up to 0.94 leads to a similar sequence. (Increasing $\theta$ further would not change the ordering; it would just cause some countries to fail at earlier waves.) Interestingly, Italy is in the last wave of failures in each case; this is due to its low exposure to others' debts. Its GDP is not particularly strong, but it does not hold much of the debt of the other countries, with the exceptions of France and Germany.

Clearly the above exercise is based on rough numbers, ad hoc estimates for the default thresholds, and a closed (six-country) world. Nonetheless, it illustrates the simplicity of the approach and makes it clear that much more accurate simulations could be run with access to precise cross-holdings data, default costs, and thresholds. ${ }^{63}$

We reemphasize that the cascades are (hopefully) off the equilibrium path, but that understanding the dependency matrix and the hierarchical structure of potential cascades can improve policy interventions.

\section{Concluding Remarks}

Based on a simple model of cross-holdings among organizations that allows discontinuities in values, we have examined cascades in financial networks. We have highlighted several important features. First, diversification and integration are usefully distinguished as they have different effects on financial contagions. Second, both diversification and integration entail trade-offs in how they affect contagion. These trade-offs result in nonmonotonic effects where middle ranges are the most dangerous with respect to cascades of failures. The trade-offs can also be related to important realistic aspects of a network, such as its core-periphery and segregation structure.

\footnotetext{
${ }^{62}$ Those values are calculated in the same way as the values above, with the pector coming from 2008 GDP values instead of 2011 and again normalized by setting Portugal's 2011 GDP to 1.

${ }^{63}$ Of course, a linear cross-holdings structure is also an important simplification. A further refinement would involve modeling the holdings in greater detail, and solving for the ultimate dependencies of organizations on assets (analogous to computing the A matrix) in that more complicated world.
} 
Table 1-Hierarchies of Cascades in the Best-Case Equilibrium Algorithm, as a Function of the FaILURe Threshold $\theta$

\begin{tabular}{lcccc}
\hline \hline Value of $\theta$ & 0.9 & 0.93 & 0.935 & 0.94 \\
\hline First failure & Greece & Greece & Greece & Greece \\
Second failure & & Portugal & Portugal, Spain \\
Third failure & & Spain & France, Germany \\
Fourth failure & & France & Italy \\
Fifth failure & & Germany, Italy & \\
\hline
\end{tabular}

Source: Authors' calculations

A fully endogenous study of the network of cross-holdings and of asset holdings is a natural next step. ${ }^{64}$ We illustrate some moral hazard issues in online Appendix Section 3: organizations can have incentives to affect both bankruptcy costs and thresholds in socially inefficient ways. These considerations suggest that endogenizing the basic structures of our model will be delicate and that a simple general equilibrium approach will not suffice. This presents interesting challenges for future research.

The approach we have outlined could be used to inform policy. For example, counterfactual scenarios can be run using the algorithm. To determine the marginal effect of saving a set of organizations, the failure costs of those organizations can be set to zero and the algorithm run with and without their failure costs. Such a simulation identifies a new set of organizations to fail in a cascade conditional on the intervention. This set of organizations can be compared to the set of organizations that fail under other interventions, including doing nothing. It is important to note that the aforementioned exercise must be repeated for any set of underlying asset prices that are of interest. As underlying asset prices change, the differences between organizations' values and their failure thresholds change. These changes may be highly correlated depending on the underlying asset holdings. When many organizations have similar exposures to underlying assets, they will be relatively close to their failure frontiers at the same time, and so the first (and subsequent) waves of failures may change drastically for fairly small changes in asset prices.

\section{APPENDIX: ProOFs}

\section{PROOF OF LEMMA 1:}

One representation of $\mathbf{A}$ is as the following infinite sum, known as the Neumann series:

$$
\mathbf{A}=\hat{\mathbf{C}} \sum_{p=0}^{\infty} \mathbf{C}^{p}=\hat{\mathbf{C}}+\hat{\mathbf{C}} \sum_{p=1}^{\infty} \mathbf{C}^{p}
$$

It follows that $A_{i i} \geq \hat{C}_{i i}$ and that there is equality if and only if there are no cycles involving $i$. Part (ii) can be proved by considering $\hat{\mathbf{C}}$ and $\mathbf{C}$ such that $\hat{C}_{i i}=\epsilon$ for all $i$

\footnotetext{
${ }^{64}$ For some analyses of network formation in other financial settings, see Babus (2013); Ibragimov, Jaffee, and Walden (2011); Cohen-Cole, Patacchini, and Zenou (2012); and Baral (2012). Incentives can cut in either direction, as firms have some incentives to protect themselves (e.g., Babus 2013), but might also wish to take excessively risky investments since they do not internalize the costs of others' exposures.
} 
and $C_{i j}=(1-\epsilon) /(n-1)$ for all $i$ and all $j$. Taking $\epsilon \rightarrow 0$, we have $\hat{C}_{i i} \rightarrow 0$, while A tends to the matrix with all entries equal to $1 / n$.

\section{PROOF OF PROPOSITION 1:}

As by hypothesis row $i$ of $\mathbf{A}^{\prime}$ differs from the same row of $\mathbf{A}$, after any trade there must exist a price vector $\mathbf{p}^{\prime \prime}$ within an $\epsilon$ neighborhood of $\lambda \mathbf{p}$ such that $v_{i}\left(\mathbf{p}^{\prime \prime}, \mathbf{C}^{\prime}, \mathbf{D}^{\prime} \mid \mathcal{Z}=\emptyset\right) \neq v_{i}\left(\mathbf{p}^{\prime \prime}, \mathbf{C}, \mathbf{D} \mid \mathcal{Z}=\emptyset\right)=\underline{v}_{i}$. For the proposition to be false, it must then be that, for all such choices of $\mathbf{p}^{\prime \prime}, v_{i}\left(\mathbf{p}^{\prime \prime}, \mathbf{C}^{\prime}, \mathbf{D}^{\prime} \mid \mathcal{Z}=\emptyset\right)>$ $v_{i}\left(\mathbf{p}^{\prime \prime}, \mathbf{C}, \mathbf{D} \mid \mathcal{Z}=\emptyset\right)$. Define price $\mathbf{p}^{\prime}$ such that $\frac{1}{2} \mathbf{p}^{\prime \prime}+\frac{1}{2} \mathbf{p}^{\prime}=\lambda \mathbf{p}$. As $\left\|\mathbf{p}^{\prime}-\lambda \mathbf{p}\right\|_{\infty}$ $=\left\|\mathbf{p}^{\prime \prime}-\lambda \mathbf{p}\right\|_{\infty}$ and $\mathbf{p}^{\prime \prime}$ is within an $\epsilon$ neighborhood of $\lambda \mathbf{p}, \mathbf{p}^{\prime}$ is also within an $\epsilon$ neighborhood of $\lambda \mathbf{p}$.

By the linearity of organizations' values, absent any failure, and as the trade was fair,

$$
\frac{1}{2} v_{i}\left(\mathbf{p}^{\prime \prime}, \mathbf{C}^{\prime}, \mathbf{D}^{\prime} \mid \mathcal{Z}=\emptyset\right)+\frac{1}{2} v_{i}\left(\mathbf{p}^{\prime}, \mathbf{C}^{\prime}, \mathbf{D}^{\prime} \mid \mathcal{Z}=\emptyset\right)=v_{i}\left(\lambda \mathbf{p}, \mathbf{C}^{\prime}, \mathbf{D}^{\prime} \mid \mathcal{Z}=\emptyset\right)=\underline{v}_{i},
$$

and

$$
\underline{v}_{i}=v_{i}(\lambda \mathbf{p}, \mathbf{C}, \mathbf{D} \mid \mathcal{Z}=\emptyset)=\frac{1}{2} v_{i}\left(\mathbf{p}^{\prime \prime}, \mathbf{C}, \mathbf{D} \mid \mathcal{Z}=\emptyset\right)+\frac{1}{2} v_{i}\left(\mathbf{p}^{\prime}, \mathbf{C}, \mathbf{D} \mid \mathcal{Z}=\emptyset\right) .
$$

Thus as $v_{i}\left(\mathbf{p}^{\prime \prime}, \mathbf{C}^{\prime}, \mathbf{D}^{\prime} \mid \mathcal{Z}=\emptyset\right)>v_{i}\left(\mathbf{p}^{\prime \prime}, \mathbf{C}, \mathbf{D} \mid \mathcal{Z}=\emptyset\right)$,

$$
v_{i}\left(\mathbf{p}^{\prime}, \mathbf{C}^{\prime}, \mathbf{D}^{\prime} \mid \mathcal{Z}=\emptyset\right)<\underline{v}_{i}<v_{i}\left(\mathbf{p}^{\prime}, \mathbf{C}, \mathbf{D} \mid \mathcal{Z}=\emptyset\right)
$$

\section{PROOF OF PROPOSITION 2:}

Recall that $\mathcal{Z}_{k}$ is the set of organizations that fail in or before hierarchy (or wave) $k$ of a cascade and let $\mathcal{Z}_{0}=\emptyset$. The value of organization $i$ then evolves with the cascade hierarchies so that

$$
v_{i}\left(\mathcal{Z}_{k-1}\right)=\sum_{j \notin \mathcal{Z}_{k-1}}^{n} A_{i j} D_{j k} p_{k}+\sum_{j \in \mathcal{Z}_{k-1}}^{n} A_{i j}\left(D_{j k} p_{k}-\beta_{j}\right)=v_{i}(\emptyset)-\sum_{j \in \mathcal{Z}_{k-1}}^{n} A_{i j} \beta_{j} .
$$

As fair trades hold constant $v_{i}(\emptyset)$, the same set of organizations must initially fail for $(\mathbf{p}, \mathbf{C}, \mathbf{D})$ and $\left(\mathbf{p}, \mathbf{C}^{\prime}, \mathbf{D}^{\prime}\right)$. The above equation shows that the value of organization $i$ given failures $\mathcal{Z}_{k-1}$ is weakly decreasing in $A_{i j}$ for all $j \neq i$ and for all cascade hierarchies $k$. This implies that holding fixed the hierarchies in which all other organizations fail, after a weak increase in $A_{i j}$ for all $i$ and all $j \neq i$, if organization $i$ failed in hierarchy $k$ it will now fail (weakly) sooner in hierarchy $k^{\prime} \leq k$; and if organization $i$ did not fail in any hierarchy it might now fail in some hierarchy.

Moreover, failures are complementary. If organization $i$ fails strictly sooner in hierarchy $k^{\prime}$, weakly more organizations will be included in all subsequent failure sets $\mathcal{Z}_{k^{\prime \prime}}$, for all $k^{\prime \prime}>k^{\prime}$. This is because more failure costs are summed over in the above equation when calculating an organization's value in each failure hierarchy.

\section{PROOF OF LEMMA 2:}

Let $\overline{\mathbf{C}}=\mathbf{G d}^{-1}$ and note that by the Neumann series we may write 


$$
\begin{aligned}
\mathbf{A} & =(1-c) \sum_{t=0}^{\infty} c^{t} \overline{\mathbf{C}}^{t} \\
\frac{\partial \mathbf{A}}{\partial c} & =(1-c) \sum_{t=1}^{\infty} t c^{t-1} \overline{\mathbf{C}}^{t}-\sum_{t=0}^{\infty} c^{t} \overline{\mathbf{C}}^{t}=-\mathbf{I}+\sum_{t=1}^{\infty}(t(1-c)-c) c^{t-1} \overline{\mathbf{C}}^{t} .
\end{aligned}
$$

Since $c<\frac{1}{2}$, every term in the summation over $t$ is nonnegative. Moreover, $c^{t-1} \overline{\mathbf{C}}^{t}$ has a strictly positive $(i, j)$ th entry whenever there is an ownership path of length $t$ from $i$ to $j$ in $\overline{\mathbf{C}}$, or equivalently in G. This shows (ii) and (iii). To verify (i), note that every column of $\mathbf{A}$ sums to 1 . Claim (iii) along with the assumption that every node in $\mathbf{G}$ has at least one neighbor shows that every column has an off-diagonal entry that strictly increases in $c$; and no off-diagonal entry decreases by (ii). So the diagonal entries strictly decrease in $c$.

\section{PROOF OF PROPOSITION 3:}

We begin with a simple lemma, whose proof can be found in Section 11 of the online Appendix.

LEMMA 3: The values $\tilde{v}_{\max }$ and $\tilde{v}_{\min }$ are upper and lower bounds, respectively, for the value of any organization.

We also introduce some terminology. If $C_{j i}>0$ there is an edge from $i$ to $j$ in the cascade network - corresponding to value flowing from $i$ to $j$. We adopt the same convention for $\mathbf{G}$ : we say there is an edge from $i$ to $j$ if $G_{j i}=1$, and define paths analogously-recall footnote 11 . That is, in this proof, we work in the network of cascade paths, rather than of ownership paths. Fixing a graph $\mathbf{G}$ and a node $i$, the fan-out of $i$, denoted $\mathcal{R}^{+}(i)$, is the set of nodes $j$ such that there is a directed path from $i$ to $j$ in $\mathbf{G}$. These are the $j$ 's that have direct or indirect cross-holdings in $i$. Throughout, $\mathbf{G}$ is drawn uniformly at random from $\mathcal{G}\left(\boldsymbol{\pi}, n_{k}\right)$, with $n_{k}$ left implicit.

If 3A(i) in Proposition 3's statement holds $(d<1)$, then by Theorem 1 of Cooper and Frieze (2004), for any $\varepsilon>0$ and large enough $k$, with probability at least $1-\varepsilon$ there are no nodes having a fan-out larger than $\varepsilon n_{k}$. Since only nodes in $\mathcal{R}^{+}(i)$ can fail following the failure of $i$, this proves that for large enough $k$, we have $f\left(\pi, n_{k}\right) \leq \varepsilon$.

Suppose 3A(ii) in the proposition's statement holds. Fix $\varepsilon>0$. Suppose that proprietary asset $i$ (belonging to organization $i$ ) is the one that is randomly selected to fail. Take any $j$ such that $G_{j i}>0$. The amount by which the value of organization $j$ falls is $A_{j i}$. By the Neumann series (equation (A1)), $A_{j i} \leq(1-c) c / \underline{d}+R_{j i}$, where $R_{j i}=(1-c)\left(\sum_{p=2}^{\infty} \mathbf{C}^{p}\right)_{j i}$ accounts for the value flowing along paths from $i$ to $j$ in $\mathbf{C}$ other than the edge from $i$ to $j$ with weight $C_{j i}$-i.e., paths of length 2 or longer. The following is proved in Section 11 of the online Appendix:

LEMMA 4: For any $\varepsilon$, if $k$ is large enough, then with probability at least $1-\varepsilon$, simultaneously for all $j$ such that $G_{j i}=1$, we have $R_{j i}=(1-c)\left(\sum_{p=2}^{\infty} \mathbf{C}^{p}\right)_{j i} \leq \varepsilon$.

By $3 \mathrm{~A}$ (ii) in the proposition's statement, and Lemma $3,(1-c) c / \underline{d}<\tilde{v}_{\min }-$ $\underline{v} \leq v_{j}-\underline{v}$. So, for small enough $\varepsilon$, a failure of $i$, which reduces $j$ 's value by at most 
$(1-c) c / \underline{d}+\varepsilon$, is not enough to cause the failure of any counterparty $j$, and so there is no contagion.

Now suppose 3B(i) and 3B(ii) hold, and again fix $\varepsilon>0$. Let $i$ be the index of the first asset to fail. By Theorems 2 and 3 of Cooper and Frieze (2004), because $d>1$, with probability at least $\varepsilon$ the node $i$ has fan-out of size at least $\varepsilon n_{k}$, for small enough $\varepsilon$ and large enough $k$. Suppose that organization $j$ has holdings in organization $i$ (i.e., $G_{j i}>0$ ), and recall that if organization $i$ fails (resulting in the devaluation of $i$ 's proprietary asset from 1 to 0 ), organization $j$ 's value will decrease by $A_{j i}$. By the Neumann series (equation $(\mathrm{A} 1)) A_{j i} \geq \frac{c(1-c)}{\bar{d}}$, deterministically. ${ }^{65}$ Organization $j$ will therefore fail, following the failure of organization $i$ if:

$$
v_{i}-\frac{c(1-c)}{\bar{d}}<\underline{v},
$$

which is guaranteed by $\bar{d}<\frac{c(1-c)}{\tilde{v}_{\max }-\underline{v}}$. This argument applies again to all the neighbors of $j$ once it fails; iterating this argument, we find that the whole set $\mathcal{R}^{+}(i)$ fails. Thus, in the event (probability $\geq \varepsilon$ ) that node $i$ has fan-out of size at least $\varepsilon n_{k}$, at least $\varepsilon n_{k}$ nodes fail, which establishes that $f\left(\pi, n_{k}\right) \geq \varepsilon^{2}$ for large enough $k$.

This completes the proof of the proposition.

\section{REFERENCES}

Acemoglu, Daron, Vasco M. Carvalho, Asuman Ozdaglar, and Alireza Tahbaz-Salehi. 2012. "The Network Origins of Aggregate Fluctuations.” Econometrica 80 (5): 1977-2016.

Acemoglu, Daron, Asuman Ozdaglar, and Alireza Tahbaz-Salehi. 2012. "Systemic Risk and Stability in Financial Networks." Unpublished.

Allen, Franklin, and Ana Babus. 2009. "Networks in Finance." In The Network Challenge: Strategy, Profit, and Risk in an Interlinked World, edited by Paul R. Kleindorfer and Yoram Wind, 367-82. Philadelphia: Wharton School Publishing.

Allen, Franklin, Ana Babus, and Elena Carletti. 2012. "Asset Commonality, Debt Maturity and Systemic Risk.” Journal of Financial Economics 104 (3): 519-34.

Allen, Franklin, and Douglas Gale. 2000. "Financial Contagion." Journal of Political Economy 108 (1): $1-33$.

Alvarez, Fernando, and Gadi Barlevy. 2013. "Mandatory Disclosure and Financial Contagion." Unpublished.

Babus, Ana. 2013. "The Formation of Financial Networks.” Unpublished.

Bank for International Settlements. 2012. "BIS Quarterly Review, June 2012: Detailed Tables." http:// www.bis.org/publ/qtrpdf/r_qa1206.pdf (accessed August 28, 2014).

Baral, Pallavi. 2012. "Strategic Behavior and Endogenous Risk of Contagion in a Financial Network: A Network Formation Game." Unpublished.

-Billio, Monica, Mila Getmansky, Andrew W. Lo, and Loriana Pelizzon. 2012. "Econometric Measures of Connectedness and Systemic Risk in the Finance and Insurance Sectors.” Journal of Financial Economics 104 (3): 535-59.

Blume, Lawrence, David Easley, Jon Kleinberg, Robert Kleinberg, and Éva Tardos. 2011. "Which Networks Are Least Susceptible to Cascading Failures?” In 2011 IEEE 52nd Annual Symposium on Foundations of Computer Science, edited by Rafail Ostrovsky, 393-402. Piscataway, NJ: IEEE.

Brioschi, Francesco, Luigi Buzzacchi, and Massimo M. Colombo. 1989. "Risk Capital Financing and the Separation of Ownership and Control in Business Groups." Journal of Banking and Finance 13 (4-5): 747-72.

\footnotetext{
${ }^{65}$ This lower bound on $A_{j i}$ can be found by considering only the direct effect of $j$ 's cross-holdings in $i$ and not any further feedbacks.
} 
Cabrales, Antonio, Pietro Gottardi, and Fernando Vega-Redondo. 2013. "Risk-Sharing and Contagion in Networks." Unpublished.

-Cifuentes, Rodrigo, Gianluigi Ferrucci, and Hyun Song Shin. 2005. "Liquidity Risk and Contagion." Journal of the European Economic Association 3 (2-3): 556-66.

Cohen-Cole, Ethan, Eleonora Patacchini, and Yves Zenou. 2012. "Systemic Risk and Network Formation in the Interbank Market." Unpublished.

-Cooper, Colin, and Alan Frieze. 2004. "The Size of the Largest Strongly Connected Component of a Random Digraph with a Given Degree Sequence." Combinatorics, Probability, and Computing 13 (3): 319-37.

Davydenko, Sergei A., Ilya A. Strebulaev, and Xiaofei Zhao. 2012. "A Market-Based Study of the Cost of Default." Rock Center for Corporate Governance at Stanford University Working Paper 124.

Demange, Gabrielle. 2012. "Contagion in Financial Networks: A Threat Index.” Paris School of Economics Working Paper 2012-02.

Dette, Tilman, Scott Pauls, and Daniel N. Rockmore. 2011. "Robustness and Contagion in the International Financial Network." Arxiv 1104.4249v2 [q-fin.GN].

Diamond, Douglas W., and Philip H. Dybvig. 1983. "Bank Runs, Deposit Insurance, and Liquidity." Journal of Political Economy 91 (3): 401-19.

Diebold, Francis X., and Kamil Yilmaz. 2011. "On the Network Topology of Variance Decompositions: Measuring the Connectedness of Financial Firms.” Penn Institute for Economic Research Working Paper 11-031.

- Eisenberg, Larry, and Thomas H. Noe. 2001. "Systemic Risk in Financial Systems.” Management Science 47 (2): 236-49.

-Elsinger, Helmut, Alfred Lehar, and Martin Summer. 2006. "Risk Assessment for Banking Systems." Management Science 52 (9): 1301-14.

Fedenia, Mark, James E. Hodder, and Alexander J. Triantis. 1994. "Cross-Holdings: Estimation Issues, Biases, and Distortions." Review of Financial Studies 7 (1): 61-96.

-French, Kenneth R., and James M. Poterba. 1991. "Were Japanese Stock Pries Too High?” Journal of Financial Economics 29 (2): 337-63.

Gai, Prasanna, Andrew Haldane, and Sujit Kapadia. 2011. "Complexity, Concentration and Contagion." Journal of Monetary Economics 58 (5): 453-70.

Gai, Prasanna, and Sujit Kapadia. 2010. "Contagion in Financial Networks." Proceedings of the Royal Society A 466 (2120): 2401-23.

Glasserman, Paul, and H. Peyton Young. 2013. "How Likely is Contagion in Financial Networks?' Unpublished.

Gofman, Michael. 2013. "Efficiency and Stability of a Financial Architecture with Too Interconnected to Fail Institutions." Unpublished.

Gouriéroux, Christian S., Jean-Cyprien Héam, and Alain Monfort. 2012. "Bilateral Exposures and Systemic Solvency Risk.” Canadian Journal of Economics 45 (4): 1273-1309.

Greenwood, Robin, Augustin Landier, and David Thesmar. 2012. "Vulnerable Banks." National Bureau of Economic Research Working Paper 18537.

Ibragimov, Rustam, Dwight Jaffee, and Johan Walden. 2011. "Diversification Disasters." Journal of Financial Economics 99 (2): 333-48.

Jackson, Matthew O. 2008. Social and Economic Networks. Princeton, NJ: Princeton University Press.

-James, Christopher. 1991. "The Losses Realized in Bank Failures." The Journal of Finance 46 (4): 1223-42.

Kiyotaki, Nobuhiro, and John Moore. 1997. “Credit Cycles.” Journal of Political Economy 105 (2): 211-48.

Leitner, Yaron. 2005. "Financial Networks: Contagion, Commitment, and Private Sector Bailouts." Journal of Finance 60 (6): 2925-53.

Leontief, Wassily W. 1951. Input-Output Economics. New York: Oxford University Press.

Lorenz, Jan, Stefano Battiston, and Frank Schweitzer. 2009. "Systemic Risk in a Unifying Framework for Cascading Processes on Networks.” European Physical Journal B 71 (4): 441-60.

Meyer, Carl D. 2000. Matrix Analysis and Applied Linear Algebra. Philadelphia: SIAM.

Newman, Mark E. J. 2010. Networks: An Introduction. New York: Oxford University Press.

Reinhart, Carmen M., and Kenneth S. Rogoff. 2011. "The Forgotten History of Domestic Debt." The Economic Journal 121 (552): 319-50.

- Rochet, Jean-Charles, and Jean Tirole. 1996. "Interbank Lending and Systemic Risk." Journal of Money, Credit and Banking 28 (4): 733-62.

- Rogers, Chris, and Luitgard Veraart 2013. "Failure and Rescue in an Interbank Network." Management Science 59 (4): 882-98. 
Shaffer, Sherrill. 1994. "Pooling Intensifies Joint Failure Risk." Research in Financial Services (6): 249-80.

-Soramäki, Kimmo, Morten L. Bech, Jeffrey Arnold, Robert J. Glass, and Walter E. Beyeler. 2007. "The Topology of Interbank Payment Flows." Physica A 379 (1): 317-33.

-Upper, Christian. 2011. "Simulation Methods to Assess the Danger of Contagion in Interbank Markets." Journal of Financial Stability 7 (3): 111-25.

-Upper, Christian, and Andreas Worms. 2004. "Estimating Bilateral Exposures in the German Interbank Market: Is There a Danger of Contagion?” European Economic Review 48 (4): 827-49.

-Vives, Xavier. 1990. "Nash Equilibrium with Strategic Complementarities." Journal of Mathematical Economics 19 (3): 305-21.

-Wagner, Wolf. 2010. "Diversification at Financial Institutions and Systemic Crises." Journal of Financial Intermediation 19 (3): 373-86. 